Review

\title{
Dynamic Behavior of Li in Solid-State Li-Ion Batteries Studied using MeV Ion Beam Analysis Techniques
}

\author{
Kenji Morita ${ }^{1,}{ }^{*}$, Bun Tsuchiya ${ }^{2}$
}

1. Nagoya Industrial Science Research Institute, 1-13 Yotsuya-tori, Chikusa-ku, Japan; E-Mail: rse51151@nifty.com

2. Meijo University, 501 Shiogamaguchi, Tenpaku-ku, Japan; E-Mail: btsuchiya@meijo-u.ac.jp

* Correspondence: Kenji Morita; E-mail: rse51151@nifty.com

Academic Editor: Ahamed Irshad

Special Issue: Batteries: Past, Present and Future

Journal of Energy and Power Technology

2021, volume 3, issue 2

doi:10.21926/jept.2102029
Received: March 26, 2021

Accepted: June 01, 2021

Published: June 24, 2021

\section{Abstract}

In this review, various studies on the Li depth profiles of metal/electrolyte/metal capacitors and batteries of $\mathrm{Au} / \mathrm{LCO} / \mathrm{LATP} / \mathrm{Pt}$, LCO/LiPON/Si, and LMO/LiPON/NbO with different metal electrodes at both sides (by bias; $L C O=L i C_{0 O}, \quad L A T P=\mathrm{Li}_{3.1} \mathrm{Al}_{0.86} \mathrm{Ti}_{1.14} \mathrm{Ge}_{1.27} \mathrm{P}_{1.73} \mathrm{O}_{12}$, $\mathrm{LMO}=\mathrm{LiMn}_{2} \mathrm{O}_{4}, \mathrm{NbO}=\mathrm{Nb}_{2} \mathrm{O}_{5}$ ) using the in-situ reflection ERD (ERD) technique with $9 \mathrm{MeV} \mathrm{O}^{+4}$ ion beam and transmission ERD (TERD) technique with $5 \mathrm{MeV} \mathrm{He}^{+2}$ ion beam, respectively, are described. For capacitors, the transport fraction of Li-ion in the electrolyte is less than unity. The $\mathrm{Li}$ atoms diffuse in the direction opposite to the ion. It has been shown that the batteries are rechargeable. On the other hand, it is observed that an anomalous over-charging takes place when the batteries are over-biased (Si/LiPON/LCO and LMO/LiPON/NbO), and strong reactions of $\mathrm{Li}$ with the metal electrodes take place under these conditions. The anomaly observed is explained in terms of the imbalance in the capacities of $\mathrm{Li}$ in anode and cathode, which can be attributed to the sizeable amounts of hydrogen present as an impurity during the fabrication of the battery. This is because hydrogen can potentially reduce the capacity of $\mathrm{Li}$ in both anode and cathode. The reactions of Li with metal electrodes are discussed in terms of the transport fraction of Li ions (less than unity) and the difference in the work functions of

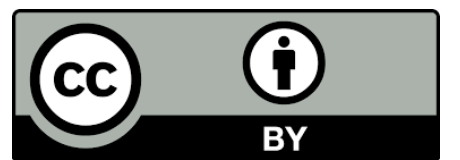


metal electrodes at both sides. Finally, it is noted that the removal of hydrogen in batteries can potentially improve safety, efficiency, and lifetime. These can be achieved by reducing the reaction of Li with metal electrodes. The recoil-scatter method in the TERD technique can measure the Li depth profile in the absence of background yields.

\section{Keywords}

Solid-state Li-ion battery; Li depth profile; MeV ion beam analysis; Li transport; charging and discharging; transport fraction of li ions; reaction of li with metal

\section{Introduction}

A variety of technologies has been developed for energy production and storage that can be used without emitting $\mathrm{CO}_{2}$. These are the safe, energy-efficient, and low-cost methods to protect the global environment. The commercially available Li-ion batteries are compact, small, and exhibit high energy density. However, they utilize an electrolyte of organic solvent, which is potentially flammable under conditions of over-charging. Flames can originate from a spark induced by a breakdown of the electrical circuit, which might be brought about by corrosion of metal electrodes. Nevertheless, its mechanism is not clear. Solid-state Li-ion batteries, in which solid non-flammable electrolyte is used for safety instead of the organic solvent, have been developed to improve its performance and establish new applications [1-3]. The problem of metal electrode corrosion is also common. Various analytical techniques such as secondary ion mass spectroscopy (SIMS), X-ray photon spectroscopy (XPS), X-ray diffraction (XRD), transmission electron microscopy (TEM), Atom Probe Tomography, Ion Beam Analysis, etc., have been used for characterizing the cathodes, electrolytes, anodes, metal electrodes, and at their interfaces to fabricate the solid-state batteries. As $\mathrm{Li}$ is highly reactive to metal, one of the most important issues is to understand the dynamic $\mathrm{Li}$ movement over the whole battery under conditions of biasing. For its establishment, the in-situ observation of total Li concentration depth distribution in the battery is of essential importance. Therefore, this review is focused on the direct and non-destructive in-situ observation of the $\mathrm{Li}$ depth profiles.

Holography-combined TEM [4], neutron depth profiling (NDP) using ${ }^{6} \mathrm{Li}$, (n, He) ${ }^{3} \mathrm{~T}$ reaction of a neutron with minor isotope ${ }^{6} \mathrm{Li}$ [5], and $\mathrm{MeV}$ ion beam analysis [6] can be potentially used for measuring the in-situ and non-destructively Li depth profiles in the Li-ion battery. The TEM technique cannot be used to detect $\mathrm{Li}$ and $\mathrm{H}$ directly, but it can be used to detect the charge depth profile of $\mathrm{Li}$ ions by combining a holography technique. NDP can also detect minor isotopes but not the other elements. Although it is very similar to the ion beam analysis from the point of view of detecting ${ }^{4} \mathrm{He}$ emitted by the nuclear reaction, its resolution in terms of energy and depth is not significantly higher compared with the ion beam analysis, which directly detects recoiled Li. The $\mathrm{MeV}$ ion beam analysis method can be used to obtain the depth profiles and atomic compositions of $\mathrm{Li}$ and $\mathrm{H}$ by reflection and transmission elastic recoil detection analysis (ERD and TERD). All elements can be analyzed by the Rutherford backscattering spectroscopy (RBS) technique except the element used as probing beam on the in-situ and direct measurement of Li depth profiles in the 
solid-state Li-ion batteries under conditions of biasing using the MeV ion beam analysis techniques [7-13].

The solid-state Li-ion battery is generally composed of stacked thin films of an anode (negative electrode), separator (electrolyte), and cathode (positive electrode) with metal electrodes at both sides, as shown in Figure 1, where the Fermi energies of these five films are different from each other. Thus, Li concentrations at each interface are enriched and depleted, and electric double layers of cathode, separator, and anodes are formed. Enrichment and depletion of $\mathrm{Li}$ at the interfaces play an important role as a barrier for Li transport in a battery. Therefore, one should understand the dynamic transport of Li under charging and discharging and evaluate and coordinate the barrier height to realize the best performance of the battery.

Thin Films Stacked Micro-Li Ion Battery

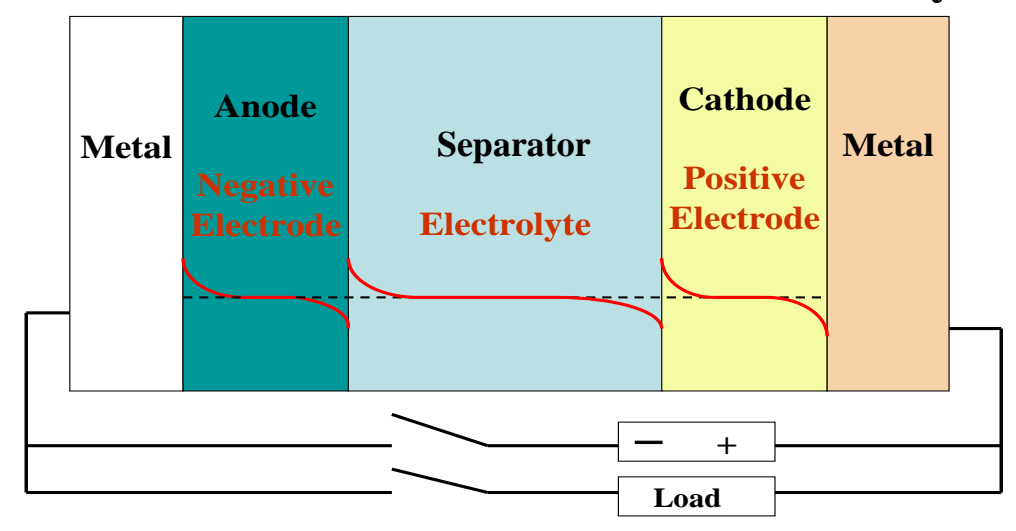

Figure 1 Schematic representation of thin films stacked micro-Li ion battery composed of an anode, separator, and cathode with metal electrodes at both sides, where the relative fractions of $\mathrm{Li}$ at their interfaces (induced as electric double layers under conditions of biasing) are shown by a red line.

In this review, the first topic of discussion (Section 3) is the experimental result obtained on Li depth profiles in capacitors of metal/electrolytes/metal without positive and negative electrodes at steady-state under biasing, using the ERD technique in the presence of the $9 \mathrm{MeV} \mathrm{O}^{+4}$ beam. The second topic describes the change in the Li profiles of the Au/LCO/LATP/Pt battery containing thick sheet electrolytes of LATP $\left(\mathrm{LCO}=\mathrm{LiCOO}_{2}, \mathrm{LATP}=\mathrm{Li}_{1+\mathrm{x}+\mathrm{y}} \mathrm{Al}_{\mathrm{y}} \mathrm{T}_{\mathrm{i2}-\mathrm{y}} \mathrm{Ge}_{\mathrm{x}} \mathrm{P}_{3-\mathrm{x}} \mathrm{O}_{12}\right)$ and Ti/NbO/LiPON/LMO/Ti battery on the Si substrate $\left(\mathrm{LiPON}=\mathrm{Li}_{3.3} \mathrm{PO}_{3.8} \mathrm{~N}_{0.2}, \quad \mathrm{LMO}=\mathrm{LiMn}_{2} \mathrm{O}_{4}\right.$, and $\left.\mathrm{NbO}=\mathrm{Nb}_{2} \mathrm{O}_{5}\right)$ under conditions of charging and discharging (Section 4). These were also measured by the RERD technique. The third topic (Section 5) deals with the change in the total Li profiles of the microbatteries of Si/LiPON/LCO and NbO/LiPON/LMO with metal electrodes at both sides prepared on self-supporting substrates. Here, the Fermi energies are different from each other. Finally, the recent results obtained from the $\mathrm{Li}$-Li and $\mathrm{He}$-Li coincidence measurements in the absence of background are also described briefly in Section 5 . These results can help detect the anomaly in the Li depth profiles of the solid-state batteries induced by over-biasing under conditions of charging and discharging. 


\section{Methods for Li Depth Profiling and Specimen Preparation}

The experiments were carried out using the B1 beam-line of $2 \mathrm{MeV}$ Tandem Accelerator at the Micro Beam Facilities of the Kyoto University. For the first and second topics, $9 \mathrm{MeV} \mathrm{O}^{+4}$ ion beam incident at an angle of $15^{\circ}$ on the surface and recoiled particles were detected using a $6 \mu \mathrm{m}$ thick $\mathrm{Al}$ absorber at a forward angle of $30^{\circ}$. The backscattered $\mathrm{O}$ ions were detected at an angle of $165^{\circ}$ to the incident direction, as shown in Figure 2 using the ERD (or ERDA) technique [14]. The final topic deals with micro-Li ion batteries (Section 5). The $5 \mathrm{MeV} \mathrm{He}^{+2}$ ion beam was normally incident on the substrate surface, and recoiled particles were detected, in the absence or the presence of the 0.8 $\mu \mathrm{m}$-thick Al absorber at a forward angle of $40^{\circ}, 45^{\circ}$, or $50^{\circ}$. The backscattered He ions were detected at an angle of $165^{\circ}$, as shown in Figure 3 , using the TERD technique.

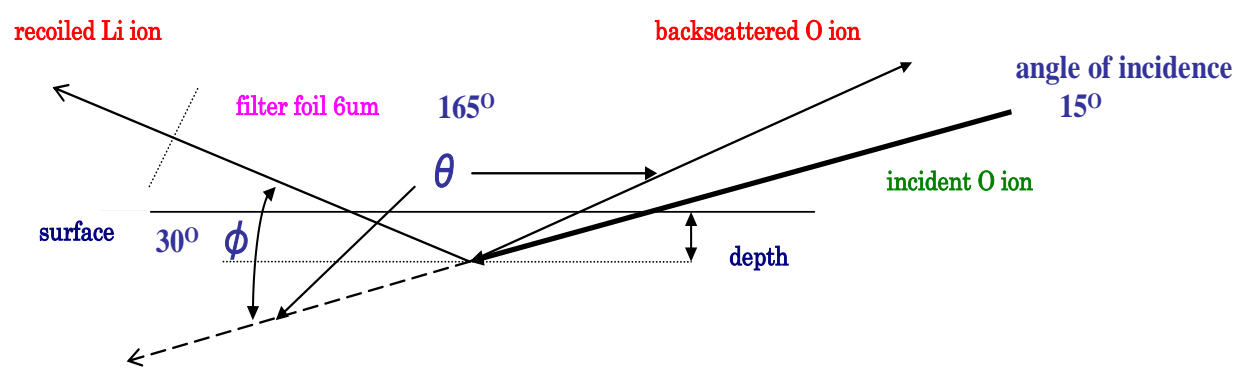

Figure 2 Geometrical schematic representation of the RERD and RBS measurement methods in the presence of the $9 \mathrm{MeV} \mathrm{O}^{+4}$ ion beam.

\section{Ni/Si/LiPON/LCO/Pt/Ti Battery on SiN Substrate}

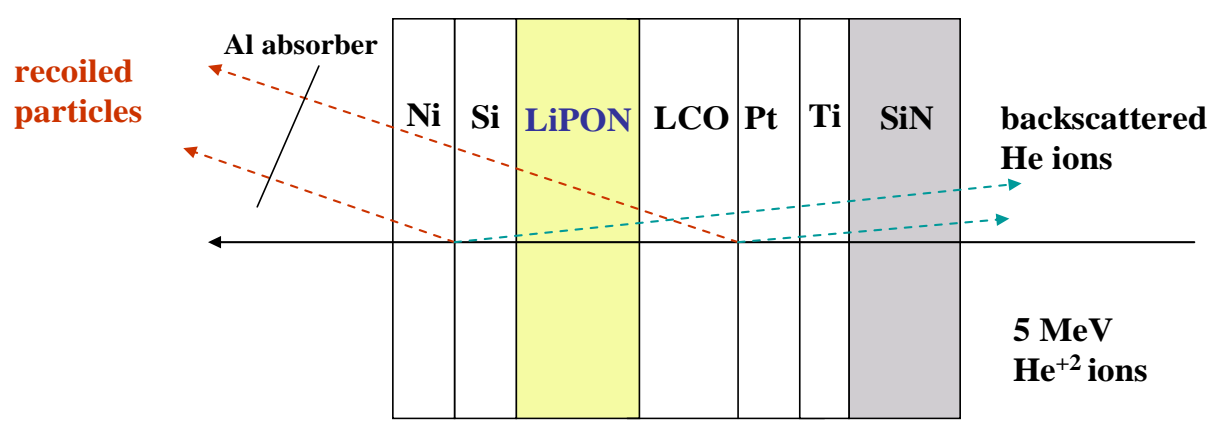

Figure 3 Geometrical schematic representation of the TERD and RBS measurement processes using the TERD technique in the presence of the $5 \mathrm{MeV} \mathrm{He}^{+2}$ ion beam.

The specimens used in the first topic (Section 3) were $\mathrm{Cu} / \mathrm{LiPON} / \mathrm{Cu}, \mathrm{Al} / \mathrm{LiPON} / \mathrm{Al}$, and Au/LATP/Au capacitors. The LiPON capacitors were prepared by depositing, in order, Ti buffer layers and films of $\mathrm{Cu}$ (or, $\mathrm{Al}$ ), $\mathrm{LiPON}$, and $\mathrm{Cu}$ (or, $\mathrm{Al}$ ) on $\mathrm{SiO}_{2}$ glass following the magnetron sputtering method [15]. The LATP capacitor was prepared by depositing Au on both surfaces of the $150 \mu \mathrm{m}$ thick LATP (commercial OHRA sheet). The film of LCO was deposited using the laser ablation method under an atmosphere of $\mathrm{O}_{2}$ [15]. The specimen used in the second topic (Section 4) was an $\mathrm{Au} / \mathrm{LCO} / \mathrm{LATP} / \mathrm{Pt}$ battery, where the negative electrode was formed using LATP and Pt. The specimens in the final topic (Section 5 ) were batteries with thin electrolyte prepared on thin selfsupporting films of Al, SiN, and Ti; Si/LiPON/LCO with pair metal electrodes of $\mathrm{Au}-\mathrm{Au} / \mathrm{Al}, \mathrm{Ni}-\mathrm{Pt} / \mathrm{Ti}$, 
and $\mathrm{NbO} / \mathrm{LiPON} / \mathrm{MLO}$ with pair electrodes of Ti-Al and Ti-Ti [16]. Large amounts of hydrogen were detected in the anodes and cathodes of these specimens and at their interfaces. Thicknesses of thin films and the composition of LATP were determined using the RBS technique in the presence of 3.55.0 $\mathrm{MeV} \mathrm{He}^{+}$and $9 \mathrm{MeV} \mathrm{O}^{+4}$ ion beams, respectively, as the deposition equipment was not provided with a thickness monitor. The composition of LATP was determined to be $y=0.84$ and $x=1.27$ (namely $\mathrm{Li}_{3.1} \mathrm{Al}_{0.84} \mathrm{Ti}_{1.16} \mathrm{Ge}_{1.27} \mathrm{P}_{1.73} \mathrm{O}_{12}$ ) using the RBS technique in the presence of the $9 \mathrm{MeV} \mathrm{O}^{+4}$ ions using the standard technique of ion beam analysis [17].

Depth profiles of the $\mathrm{Li}$ in the capacitor under conditions of biasing the bottom metal electrode at $-3 \mathrm{~V} \sim+3 \mathrm{~V}$ to the top metal electrode at earth potential were simultaneously measured using the ERD and RBS techniques. RBS was also used to monitor the irradiation dose of the incident ion beam, and the specimen was vertically displaced at each measurement to minimize the radiation effects of probing $9 \mathrm{MeV} \mathrm{O}^{+4}$ ions, as they are accumulated during repeated irradiations cycles [6].

\section{Results on Li Depth Profiles of Metal/Electrolyte/Metal Capacitors under Biasing by ERD}

Typical RBS and ERD spectra of the $9 \mathrm{MeV} \mathrm{O}^{+4}$ ion beam from the $\mathrm{Cu} / \mathrm{LiPON} / \mathrm{Cu} / \mathrm{Ti} / \mathrm{SiO}_{2}$ specimen biased at $0,+3 \mathrm{~V}$, and $-3 \mathrm{~V}$ between the electrodes of $\mathrm{Cu}$ and $\mathrm{Cu} / \mathrm{Ti}$ at earth potentials are shown in Figure 4 and Figure 5 [10]. The ERD spectra in Figure 5 are normalized by the area of Cu peaks in the RBS spectra in Figure 4. Peaks at 370 - 430 channels in Figure 5 are attributed to the $\mathrm{H}$ impurity present in LiPON during its deposition. The $\mathrm{Cu}$ peak at $+3 \mathrm{~V}$ of the RBS spectrum is seen to shift toward the lower channels to those at no bias ( $-3 \mathrm{~V}$; Figure 4). The ERD spectrum at $+3 \mathrm{~V}$ shows a new peak at 830 channels and a significant increase in the $\mathrm{H}$ peak at 430 channels, which corresponds to the hydrogens adsorbed on the Cu surface. Both results can be reasonably explained in terms of the segregation of $\mathrm{Li}$ on the surface due to biasing at $+3 \mathrm{~V}$ and the increase in the rate of adsorption of $\mathrm{H}$ due to chemical reaction between $\mathrm{Li}$ and $\mathrm{H}_{2} \mathrm{O}$ adsorbed on the surface. Such results have been, for the first time, observed by the simultaneous TERD and RBS measurements. The biasing conditions used ( $+3 \mathrm{~V})$ might be too high for the capacitor under study. The process of $\mathrm{Li}$ segregation suggests that there is a possibility of its leakage from the battery under such an anomalous condition.

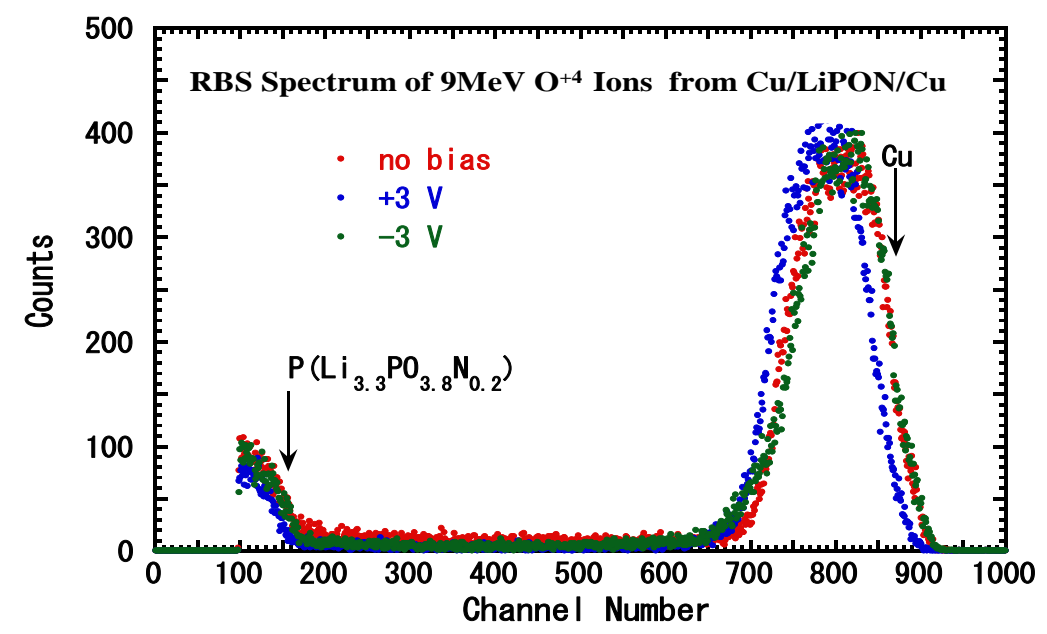

Figure 4 RBS spectral profiles of the $9 \mathrm{MeV} \mathrm{O}^{+4}$ beam emitted from $\mathrm{Cu} / \mathrm{LiPON} / \mathrm{Cu}$ biased at 0, $3 \mathrm{~V}$, and $-3 \mathrm{~V}$ [10]. 


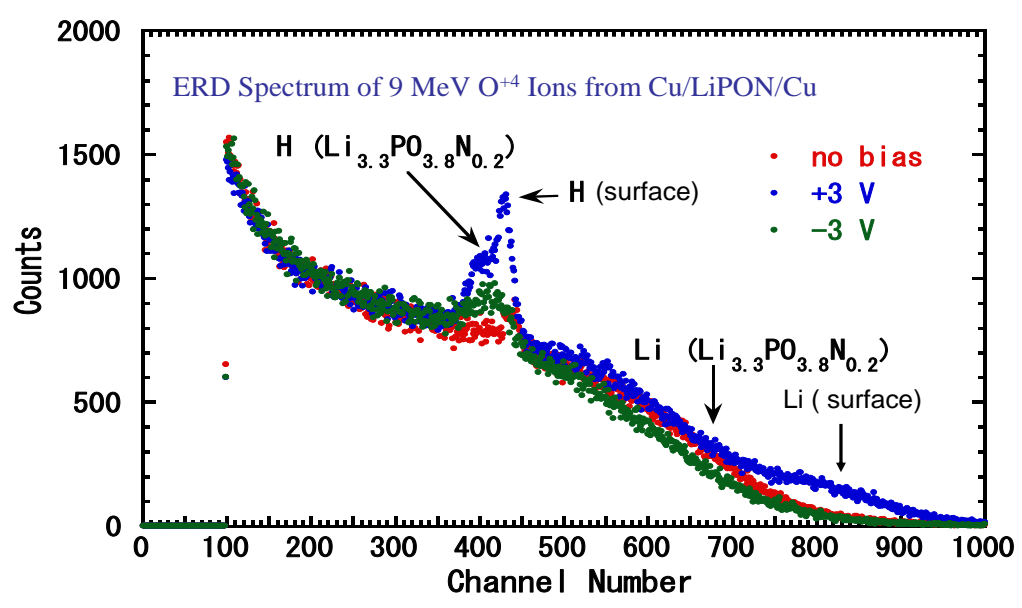

Figure 5 ERD spectral profiles of the $9 \mathrm{MeV} \mathrm{O}^{+4}$ beam emitted from $\mathrm{Cu} / \mathrm{LiPON} / \mathrm{Cu}$ biased at $0,3 \vee$, and $-3 \vee[10]$.

To closely inspect the changes in the Li depth profiles under conditions of biasing, the ratios of the ERD spectral profiles recorded under conditions of biasing and non-biasing, for $\mathrm{Cu} / \mathrm{LiPON} / \mathrm{Cu}$, $\mathrm{Al} / \mathrm{LiPON} / \mathrm{Al}$, and Au/LATP/Au, are shown in Figure 6, Figure 7, and Figure 8, respectively. The solid and dotted lines are the eye guides, and the horizontal axis represents the electrolyte depth from the interface, estimated using the stopping power of the specimen in the absence of biasing conditions and unity. The vertical axis represents the Li concentration in the absence of biasing conditions $(0 \mathrm{~V})$. It is seen from these figures that the change in the Li depth profiles is extended to approximately $170-200 \mathrm{~nm}$ due to biasing at $\pm 3 \mathrm{~V}$. This can potentially be an overestimation.

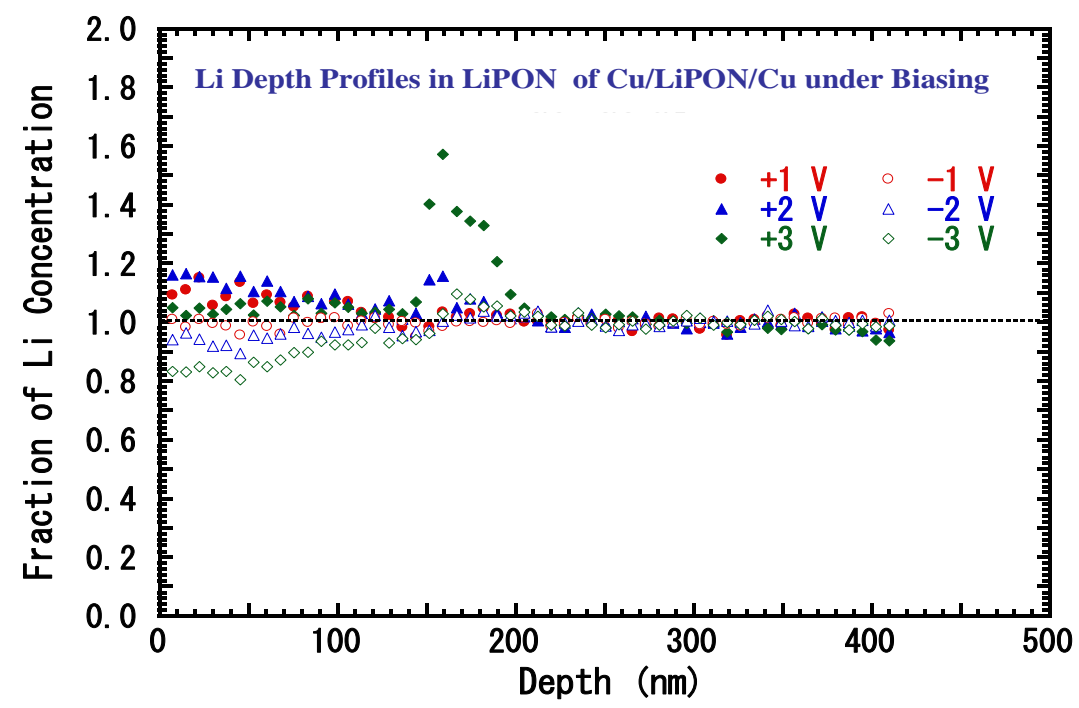

Figure 6 Change in the $\mathrm{Li}$ depth profiles of $\mathrm{Cu} / \mathrm{LiPON} / \mathrm{Cu}$ on $\mathrm{Ti} / \mathrm{SiO}_{2}$ induced by biasing [10].

The overestimation can be attributed to the large energy straggling of the incident $O$ ions passing through the specimen and recoiled $\mathrm{Li}$ ions in the $6 \mu \mathrm{m}$-thick $\mathrm{Al}$ absorber. The variation in the $\mathrm{Li}$ concentration in $\mathrm{Cu} / \mathrm{LiPON} / \mathrm{Cu}$ is lesser than that in Au/LATP/Au, although the $3.4 \mu \mathrm{m}$-thick LiPON is much thinner than LATP (150 $\mu \mathrm{m}$ thick). The Li depth profiles in Cu/LiPON/Cu and Al/LiPON/Al do not change at $-1 \mathrm{~V}$. On the other hand, the variation in Au/PATP/Au at $-1 \mathrm{~V}$ is significantly larger than 
those at $+1 \mathrm{~V}$. The latter result seems to indicate that the Li depth profile becomes flat when the bias is quite weak (several tenths of $\mathrm{V}$ ). This indicates that the Li fraction in the electrolyte at $0 \mathrm{~V}$ is enriched at the interface of $\mathrm{Cu} / \mathrm{LiPON}$ and $\mathrm{Al} / \mathrm{LiPON}$. It gets depleted or is almost flat at the interface of Au/LATP.

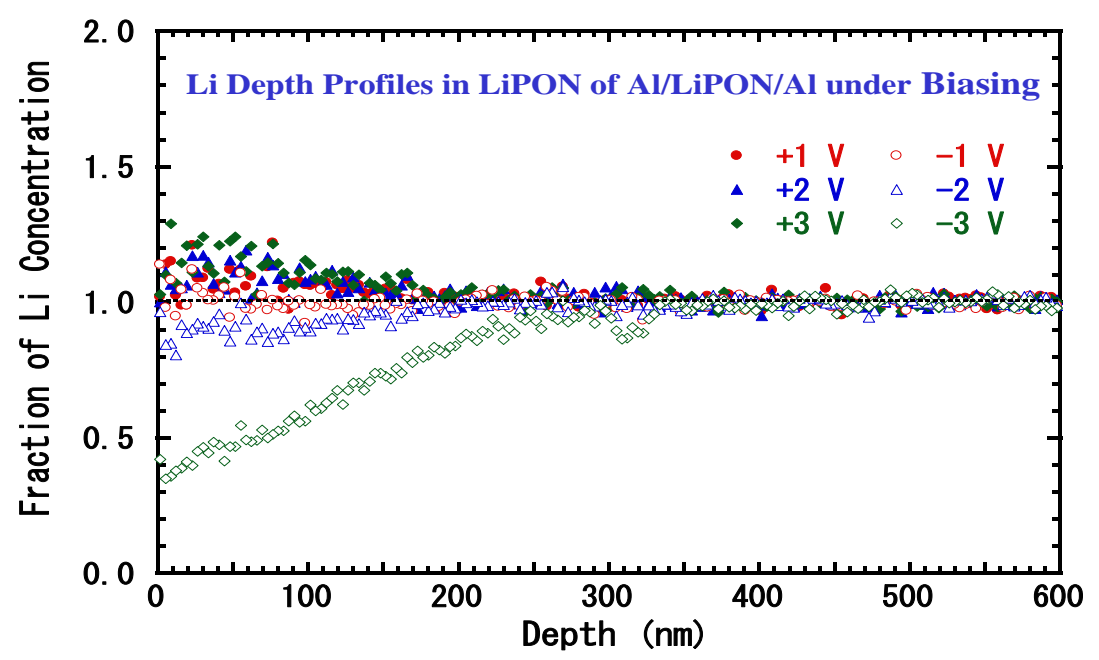

Figure 7 Change in the Li depth profiles of $\mathrm{Al} / \mathrm{LiPON} / \mathrm{Al}$ on $\mathrm{Ti} / \mathrm{SiO}_{2}$ induced by biasing [10].

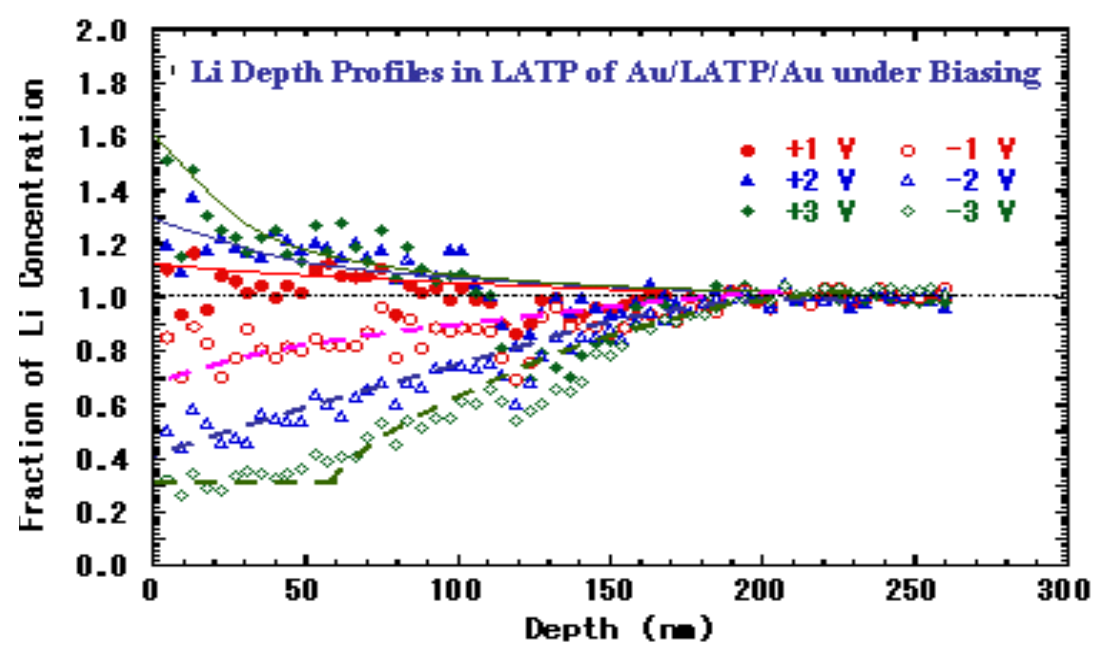

Figure 8 Change in the Li depth profile of Au/LATP/Au induced by biasing [10].

It is generally accepted that when the capacitor fabricated with metal/dielectric/metal is biased, the charge supplied by biasing is stored as uniformly distributed dipoles in the dielectric. In the capacitor fabricated with the Li-electrolyte, the charge is stored as depth profiles of the Li enriched surface. The charge gets depleted near the interfaces of both the metal electrodes. The amount of space charge stored in the electrolyte is expressed as

$$
\mathrm{Q}=\mathrm{C} \cdot \mathrm{V}+\mathrm{Q}_{\mathrm{o}}
$$

where $Q_{0}$ is the residual space charge at $V=0$, and $C$ is the electrostatic capacity.

The total number of Li moved in the electrolyte near the interfaces by biasing, which was estimated from Figure 6, Figure 7, and Figure 8, are plotted as a function of voltage in Figure 9, 
where the vertical axis corresponds to the charge stored by biasing, and the colored lines are eyeguides for the corresponding data. The amount of space charge at $0 \mathrm{~V}$ is evaluated to be positive for $\mathrm{Cu} / \mathrm{LiPON} / \mathrm{Cu} / \mathrm{Ti}$ and $\mathrm{Al} / \mathrm{LiPON} / \mathrm{Al} / \mathrm{Ti}$ and almost zero for Au/LATP/Au (Figure 9 and Equation (1)). This result reflects the asymmetry of enrichment and depletion for $\mathrm{Cu} / \mathrm{LiPON} / \mathrm{Cu}$ and Al/LiPON/Al, observed in Figure 6, Figure 7, and Figure 8. This can be explained in terms of the differences in the work functions (or Fermi energies) of both the metal electrodes fabricated using $\mathrm{Cu}$ and $\mathrm{Cu} / \mathrm{Ti}$ and $\mathrm{Al}$ and $\mathrm{Al} / \mathrm{Ti}$. As the work functions of $\mathrm{Al}, \mathrm{Ti}, \mathrm{Cu}$, and $\mathrm{Au}$ are $4.1 \mathrm{eV}, 3.9 \mathrm{eV}, 4.7 \mathrm{eV}$, and $5.1 \mathrm{eV}$ from the photo-electron emission measurements, it is deduced that the Fermi energies of the top $\mathrm{Al}$ and $\mathrm{Cu}$ electrodes are lower than those of the $\mathrm{Al} / \mathrm{Ti}$ and $\mathrm{Cu} / \mathrm{Ti}$. The top interface is enriched by $\mathrm{Li}$. The result obtained when Au/LATP/Au is studied can be explained by the symmetry of its structure. The apparent asymmetry of Au/LATP/Au observed in Figure 8 is ascribed to the large movable fraction of $\mathrm{Li}$ in LATP (increased from 1 to 3.1 by doping $\mathrm{Al}$ and $\mathrm{Ge}$ into $\mathrm{LiTi}_{2} \mathrm{P}_{3} \mathrm{O}_{12}$; compared to the case of LiPON (=Li $\left.\mathrm{Li}_{3.3} \mathrm{PO}_{3.8} \mathrm{~N}_{0.2}\right)$ ).

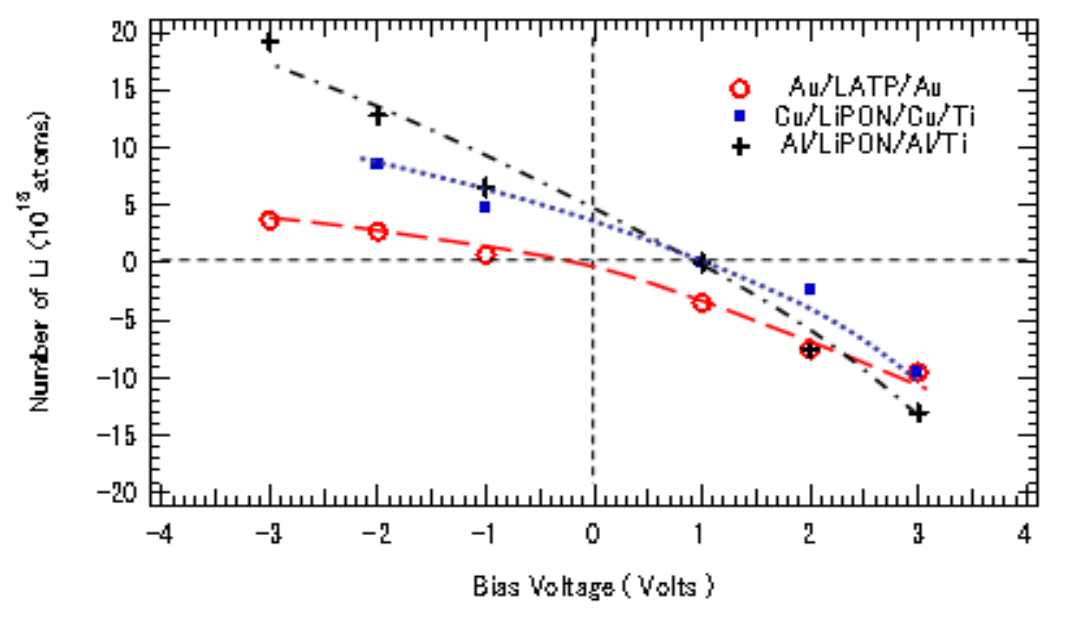

Figure 9 Voltage dependence of the numbers of Li moved by biasing which was estimated from Li depth profiles in Figure 6, Figure 7, and Figure 8 [10].

This difference in the Li-ion concentration near the interface at $0 \mathrm{~V}$ is reasonably explained in terms of the energy difference between both Fermi levels (top of conduction band) of the metal electrodes (bottom of conduction band or defect-induced donor levels in the forbidden band) and electrolyte (doped complex Li oxide) (Figure 10). The Fermi energies of both electrolyte and metal electrodes are different from each other. Thus, the electron transport at the contact interface is dependent on the energy difference, and as a result, some space charges remain. However, diffusion of the Li-ion relaxes the transport. It is presumed that the polarity of the residual space charge near the interface at $\mathrm{OV}$ is negative for $\mathrm{Cu} / \mathrm{LiPON}$ and $\mathrm{Al} / \mathrm{LiPON}$ for the asymmetric capacitor. The system is enriched by Li. On the contrary, it is neutral at Au/LATP for the symmetric one where Li may be neither depleted nor enriched. According to Figure 9, the presumption seems reasonable, although no data on the electronic structure of the doped complex Li oxide are available. 
Electrolyte

(Complex Li Oxide)

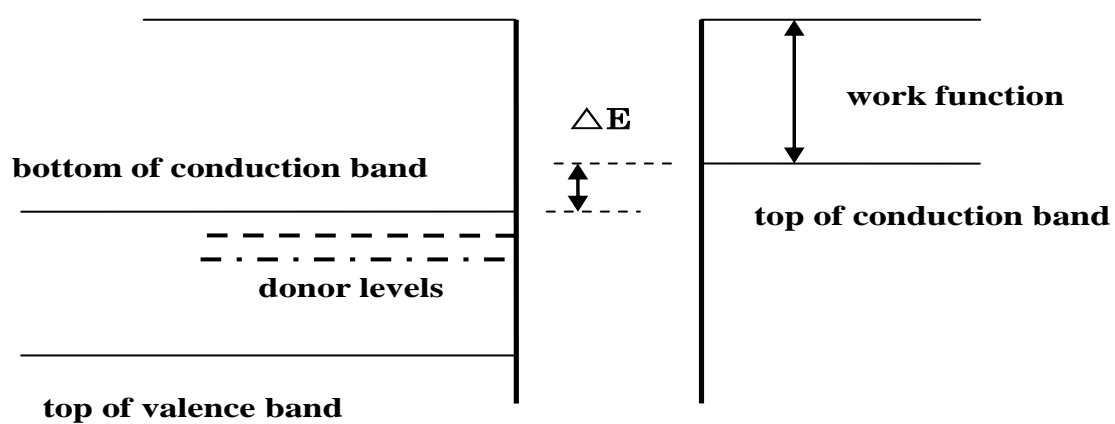

Figure $10 \mathrm{~A}$ schematic of the electronic structure of electrolyte (complex Li oxide) and metal.

The movement of $\mathrm{Li}$ in the electrolyte has been calculated according to the 2nd law of thermodynamic mechanics based on the mathematical model of space charge formation based on the first principal law [18].

Here the Li depth profile of the electrolyte at steady-state under conditions of biasing was calculated [10], based on the diffusion of Li-ion following the hopping mechanism under a gradient of potential in one dimension (Figure 11). In the figure, $x$ represents the depth from the interface, $\mathrm{n}(\mathrm{x})$ denotes the concentration of $\mathrm{Li}$, at the $\mathrm{A}, \mathrm{B}$, and $\mathrm{C}$ migration sites, $\mathrm{E}_{\mathrm{m}}$ denotes the migration energy for $\mathrm{Li}, \triangle \phi$ denotes the potential difference between the neighboring sites, and $\triangle \phi / \Delta x$ denotes the local potential gradient.

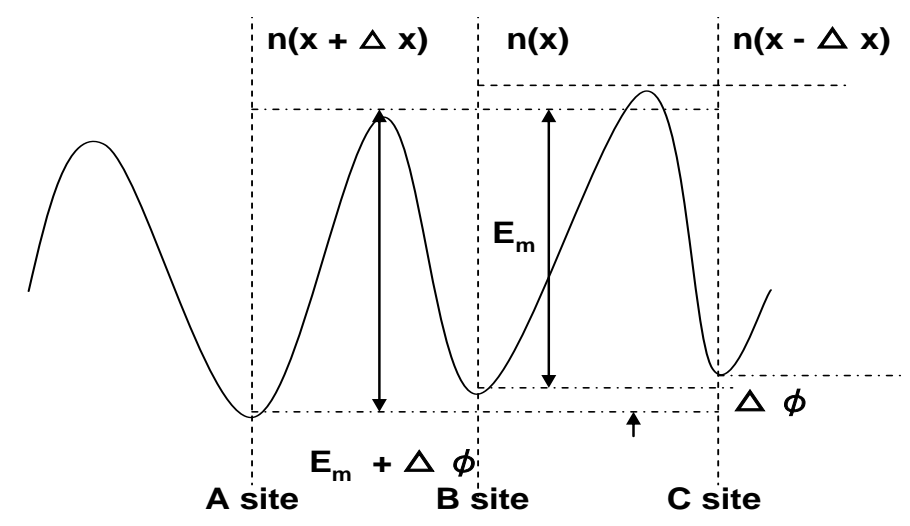

Figure 11 Schematic representation of the process of Li diffusion by the hopping mechanism under a potential gradient in one-dimensional coordination [10].

The variation in the amounts $\Delta \mathrm{n}(\mathrm{x})$ of the Li concentration via hopping among the $\mathrm{A}, \mathrm{B}$, and $\mathrm{C}$ sites is expressed as follows:

$$
\begin{gathered}
n(x)=-n(x) v_{0} \exp \left\{-\left(E_{m}-\Delta \varphi / k T\right)\right\}-n(x+\Delta x) v_{0} \exp \left\{-\left(E_{m}+\Delta \varphi\right) / k T\right\} \\
+n(x-\Delta x) v_{0} \exp -\left(E_{m}+\Delta \varphi\right) / k T+n(x+\Delta x) v_{0} \exp -\left(E_{m}-\Delta \varphi / k T\right),
\end{gathered}
$$

where $v_{0}$ is the jump frequency of Li. The current density of the Li-ions (produced due to migration) is expressed as follows: 


$$
J=D \frac{d n(x)}{d x}=-D\left\{\frac{d n(x)}{d x} \frac{\Delta \varphi}{k T}\right\}
$$

The diffusion equation for the Li-ion is calculated from Equation (2) to get the following equation:

$$
\frac{d n(x)}{d t}=-D\left\{\frac{d n(x)}{d x} \frac{d \varphi}{d x} / k T+\frac{d^{2} n(x)}{d^{2} x} \frac{\Delta \varphi}{k T}\right\}
$$

When $\mathrm{F}$ is the ionic fraction of $\mathrm{Li}$ transported under a potential gradient, the diffusion of $\mathrm{Li}$ is expressed as the sum of the ionic and neutral states:

$$
\frac{d n(x)}{d t}=D\left\{-F \frac{d \varphi}{d x} \frac{1}{k T} \frac{d n(x)}{d x}+\left(1-F-F \frac{\Delta \varphi}{k T}\right) \frac{d^{2} n(x)}{d^{2} x}\right\}
$$

The diffusion equations at steady state under conditions of biasing are easily and analytically solved

$$
n(x)=1+n_{0} \exp \left(-\frac{F}{1-F-F \Delta \varphi / k T} \frac{d \varphi}{d x} \frac{1}{k T} x\right)
$$

for negative and

$$
n(x)=1-n_{0} \exp \left(-\frac{F}{1-F-F \Delta \varphi / k T} \frac{d \varphi}{d x} \frac{1}{k T} x\right)
$$

positive bias.

It is seen from Equations (5) and (6) that the depth profile of Li in electrolyte of a capacitor at steady state condition under biasing is expressed as an exponentially decaying function of depth (from the interface). The decay depth is proportional to the inverse of the potential gradient and temperature. The depth profile is also seen to qualitatively reflect the experimental observations presented in Figure 6, Figure 7, and Figure 8. Furthermore, the ionic fraction of Li ions transported in the electrolyte should be less than $1-\Delta \varphi / \mathrm{kT}$, because the Li depth profile becomes flat if $\mathrm{F}$ $=1-\Delta \varphi / \mathrm{kT}$ in Eqs. (5) and (6). This result indicates that each of the Li depth profiles at steadystate conditions is established by balancing the diffusion of $\mathrm{Li}$ ions in the direction of flow (under the influence of a potential gradient) and Li atoms (diffusion in the counter direction; attributable to the concentration gradient).

The change in the Li depth profiles in capacitors fabricated with the Li-electrolyte was studied using the MeV ion beam analysis technique. The results were reasonably explained in terms of the hopping diffusion of the $\mathrm{Li}$ ions under the influence of a potential gradient. The transport fraction of the Li ions was $<1-\Delta \varphi / \mathrm{kT}$. 


\section{Change in the Depth Profiles of Li-Ion Batteries under Conditions of Charging and Discharging by ERD}

\subsection{Au/LCO/LATP/Pt Battery}

4.1.1 Change in the Li Depth Profiles in LCO under Conditions of charging Measured from Au Surface

The RBS and ERD energy spectra were simultaneously recorded at several stages of step biasing under charging. The typical RBS energy spectrum of the $9 \mathrm{MeV} \mathrm{O}^{+4}$ ion beam from the as-received specimen, which was measured from the top Au surface [9], is shown in Figure 12, where the energy positions of the $\mathrm{O}$ ions backscattered from $\mathrm{Au}$ at the surface and $\mathrm{Co}$ at the interfaces are indicated by differently colored arrows. The thicknesses of both $\mathrm{Au}$ and $\mathrm{LiCoO}_{2}$ were determined to be $27 \mathrm{~nm}$ and $65 \mathrm{~nm}$, respectively, from the width of their peaks. The ERD energy spectra recorded at several stages of the step biasing bottom electrode of Pt negatively are shown in Figure 13 [10]. One can see a prominent peak at around 350 channels in the ERD spectra presented in Figure 13. It was assigned to the $\mathrm{H}$ impurity involved in LCO during its preparation and adsorbed at the Au surface. The data obtained by analyzing the standard sample involving hydrogen and calculations of the energy position of the impurity $\mathrm{H}$ recoiled by $\mathrm{O}$ ions were also analyzed to arrive at this conclusion. The amount of impurity $\mathrm{H}$ was evaluated to be $\sim 5$ at.\%.

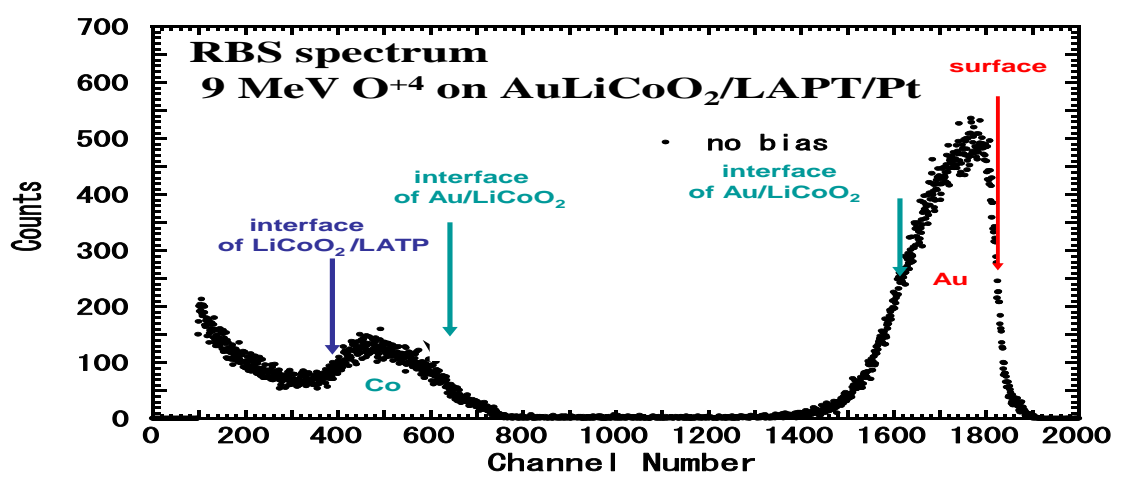

Figure 12 RBS energy spectrum of the $9 \mathrm{MeVO}^{+4}$ ion beam emitted from Au/LCO/LATP/Pt and measured from the Au surface [11].
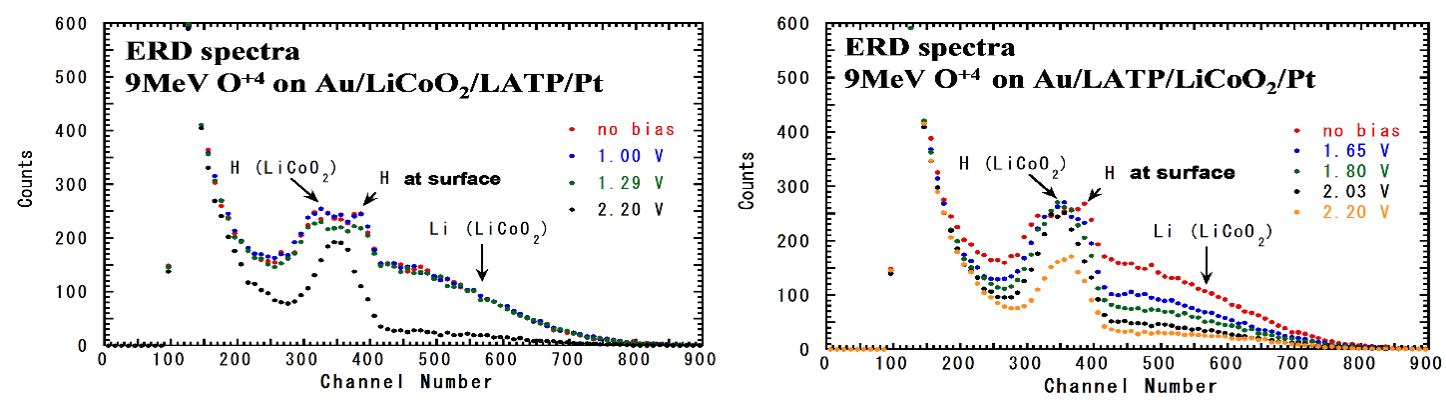

Figure $13 \mathrm{ERD}$ spectra of the $9 \mathrm{MeVO}^{+4}$ ion beam measured for the two $\mathrm{Au} / \mathrm{LiCoO}_{2} / \mathrm{LATP} / \mathrm{Pt}$ specimens under conditions of charging. Data at no bias, $1.0 \mathrm{~V}, 1.29$ $\mathrm{V}$, and $2.20 \mathrm{~V}$, are shown in the left figure and those at no bias, $1.65 \mathrm{~V}, 1.80 \mathrm{~V}, 2.13 \mathrm{~V}$, and $2.20 \mathrm{~V}$, are shown at the right figure [11]. 
It is seen from the left figure in Figure 13 that the ERD spectra at $1.0 \mathrm{~V}$ and $1.29 \mathrm{~V}$ are almost the same as no bias, which indicates that $\mathrm{Li}$ is not charged. The right figure reveals that the Li yields at the 200-800 channels decrease with an increasing bias voltage applied which indicates that charging of Li takes place. Both spectra at no bias in the left and right figures are also almost the same although the specimens are different from each other. The ratios of the biased and unbiased ERD spectra were calculated for the close inspection of the depleted fraction of the $\mathrm{Li}$ in depth (Figure 14). The interfaces of $\mathrm{LATP} / \mathrm{LiCoO}$ and $\mathrm{LiCOO}_{2} / \mathrm{Au}$ were estimated, and the results are shown using vertical dotted lines. It is seen from Figure 14 that the depletion of Li takes place uniformly over the whole depth of the $\mathrm{LiCOO}_{2}$ film. It extends into LATP (approximately $200 \mathrm{~nm}$ thick). The depth profiles of the Li depleted in LATP are very similar to those in the Au/LATP/Au capacitor under conditions of biasing shown (Figure 8; Section 3). The characteristic relation of the Li composition in $\mathrm{LiCOO}_{2}$ and bias voltage obtained from Figure 14 is plotted in Figure 15. It is seen from Figure 15 that the $\mathrm{Li}$ composition in $\mathrm{LiCoO}_{2}$ does not get reduced when the bias voltage is $\sim 1.3 \mathrm{~V}$. Hereafter, it decreases as the bias voltage increases. The characteristic relation obtained from the Li depth profiling by means of the ERD technique corresponds well with the one measured by the electrochemical method [19]. The threshold voltage ( 1.3V) for the depletion of $\mathrm{Li}$ from $\mathrm{LiCoO}_{2}$ under conditions of charging is ascribed to the enrichment of $\mathrm{Li}$ at the interface with the $\mathrm{Au}$ electrode. This can be attributed to the relaxation of the negative charge of Au with lower Fermi energy at the point of contact.

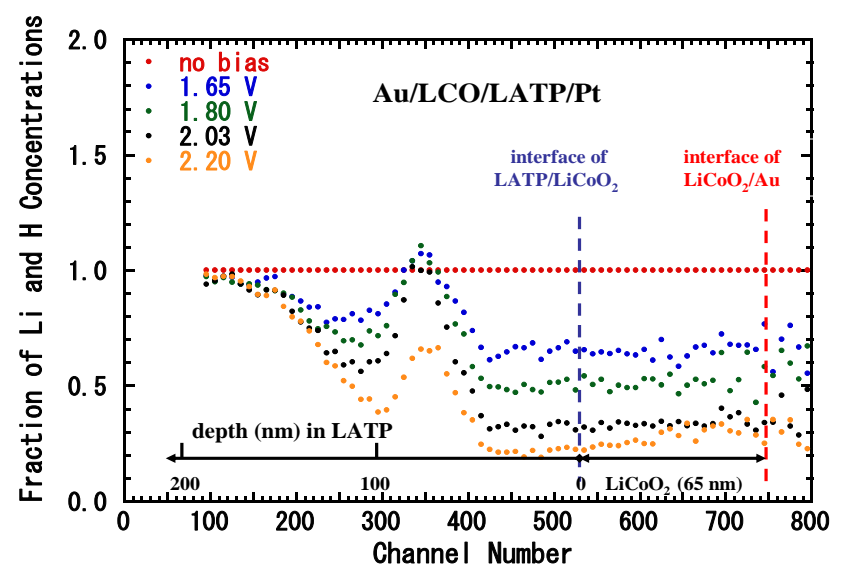

Figure 14 ERD spectral profiles under conditions of biasing normalized to no bias [11].

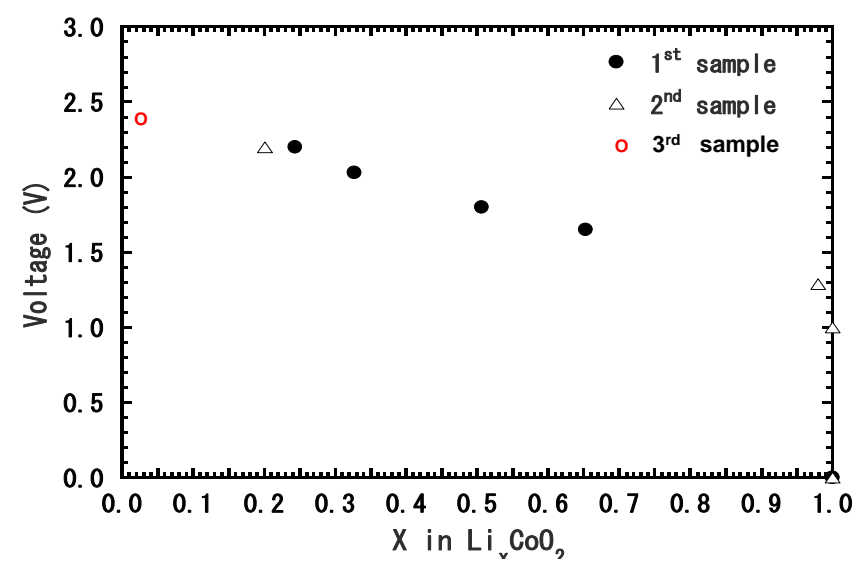

Figure 15 Relation of the Li composition in $\mathrm{LiCoO}_{2}$ with bias voltage [11]. 
4.1.2 Changes in the Li Depth Profiles Near Pt under Conditions of Charging Measured from the Pt Surface

The typical RBS energy spectrum for the as-received specimen and RRD energy spectra measured from the surface of Pt-biased negatively are shown in Figure 16 and Figure 17, respectively. In the RBS spectrum presented in Figure 16, the energy positions of the $\mathrm{O}$ ions backscattered from Pt both at the surface and interface and Ge and Ti at the interface of Pt with LATP are shown by red, green, and blue colored arrows (similar to that shown in Figure 12). The thickness of the Pt electrode was also determined to be $22 \mathrm{~nm}$ from the peak width. Figure 17 reveals that the ERD spectra of the two specimens under bias agree well with the spectra presented in Figure 13.

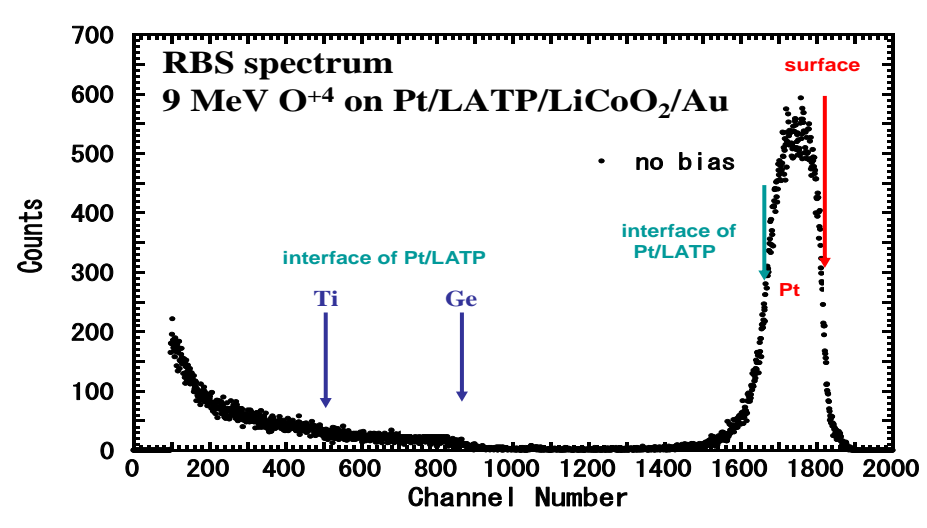

Figure 16 RBS spectrum for $\mathrm{Au} / \mathrm{LiCoO}_{2} / \mathrm{LATP} / \mathrm{Pt}[11]$.

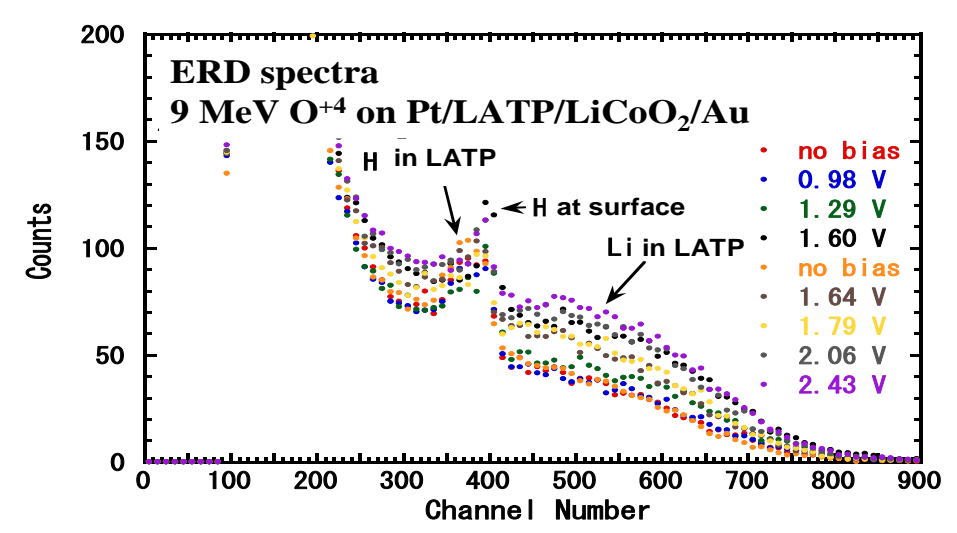

Figure 17 ERD spectral profiles of two Au/LiCoO $2 /$ LATP/Pt [11].

It is seen from the ERD spectral profiles that the yields from Li at 250 - 800 channels increase with increasing bias voltages. For close inspection of the enriched depth of $L i$, the ratios of the biased and unbiased ERD spectral profiles are shown in Figure 18, where the interface of LATP/Pt is shown using vertical dotted lines. It is seen from Figure 18 that the Li enrichment by charging is extended into LATP up to $200 \mathrm{~nm}$ from the Pt electrode. It is also noted that the Li is enriched even at the bias voltage of $1.29 \mathrm{~V}$, although $\mathrm{Li}$ in $\mathrm{LiCoO}_{2}$ gets hardly depleted (Figure 13 (left)). Although the difference in the amounts of Li enriched and depleted at both sides is negligibly small compared to the total amount of $\mathrm{Li}$ in the entire battery, the results suggest that in the absence of bias, the enrichment of $\mathrm{Li}$ at the interface of $\mathrm{Au} / \mathrm{LiCOO}_{2}$ is larger than the depletion of $\mathrm{Li}$ at the interface of LATP/Pt. The energy difference in the Fermi levels at the former interface is larger than that at the 
latter interface. Moreover, as the work function of Pt is higher than that of $\mathrm{Au}$, the Fermi level of LATP is considered to be higher than that of $\mathrm{LiCoO}_{2}$.

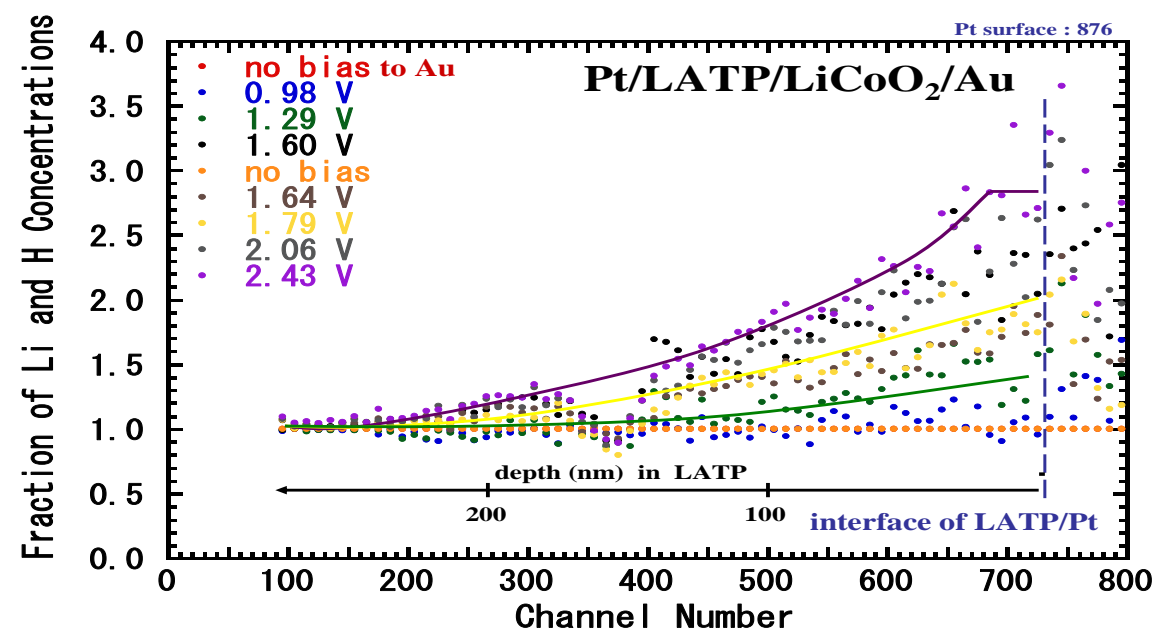

Figure 18 ERD spectral profiles at $0.98 \mathrm{~V}, 1.29 \mathrm{~V}, 1.60 \mathrm{~V}, 1.64 \mathrm{~V}, 1.79 \mathrm{~V}, 2.06 \mathrm{~V}$, and 2.43 $\checkmark$ normalized to no bias. The vertical dashed line at 735 channels represents the interface of LATP/Pt. The horizontal dotted line at unity corresponds to the Li composition in the as-prepared specimen. The solid lines are eye-guides for $2.43 \mathrm{~V}$ (violet), $1.79 \mathrm{~V}$ (yellow,) and $1.29 \mathrm{~V}$ (green). Depth scale in LATP from the interface is also indicated [11].

One can see also from Figure 18 that the enrichment factor of Li at the interface with Pt under conditions of charging seems to get saturated ( 3), even if statistical errors are involved. Another phase that is different from LATP might get precipitated because the Li enrichment factor in LATP is expected to be around 15 for $\mathrm{Li}$ in pure $\mathrm{Li}, 6.9$ for $\mathrm{Li}$ in $\mathrm{Li}_{2} \mathrm{O}$, and 3.1 for $\mathrm{Li}$ in compounds such as $\mathrm{Li}_{3} \mathrm{PO}_{4}$. The results are based on the standard composition analysis method of ERD. The phase to be precipitated should be clarified in the experiments by XRD using the thicker $\mathrm{LiCoO}_{2}$ specimen.

The depletion of $\mathrm{Li}$ in LiCoO2 and LATP at the Au side under conditions of charging is seen to correspond well with the Li enrichment in LATP at the Pt side. Charge-induced change in the entire $\mathrm{Li}$ depth profiles in the $\mathrm{Au} / \mathrm{LiCoO}_{2} / \mathrm{LATP} / \mathrm{Pt}$ battery can be imagined if Figure 14 is connected to Figure 18, where the right-side is turned left. The Li depth profiles drawn on the experimental data are shown schematically in Figure 19, where the black and green lines of the curves are imagined from the energy difference in the Fermi levels at their interfaces. These could not be experimentally observed because of limited depth resolution due to the large energy straggling of the incident $\mathrm{O}$ ions in the specimen and the recoiled Li-ions transmitted from the $6 \mu \mathrm{m}$-thick Al absorber. It is clear that Li moves due to charging from LCO to LATP near Pt, but the amounts of Li traveling between LCO and LATP cannot be confirmed because both data have been obtained using different specimens. If Li reacts with metal electrodes, as observed at the interface of LiPON/Cu in Figure 5, the Li amounts at both sides would not match. Thus, the transmission ERD technique has been developed to simultaneously determine the total Li depth profiles in the battery (Section 5). 


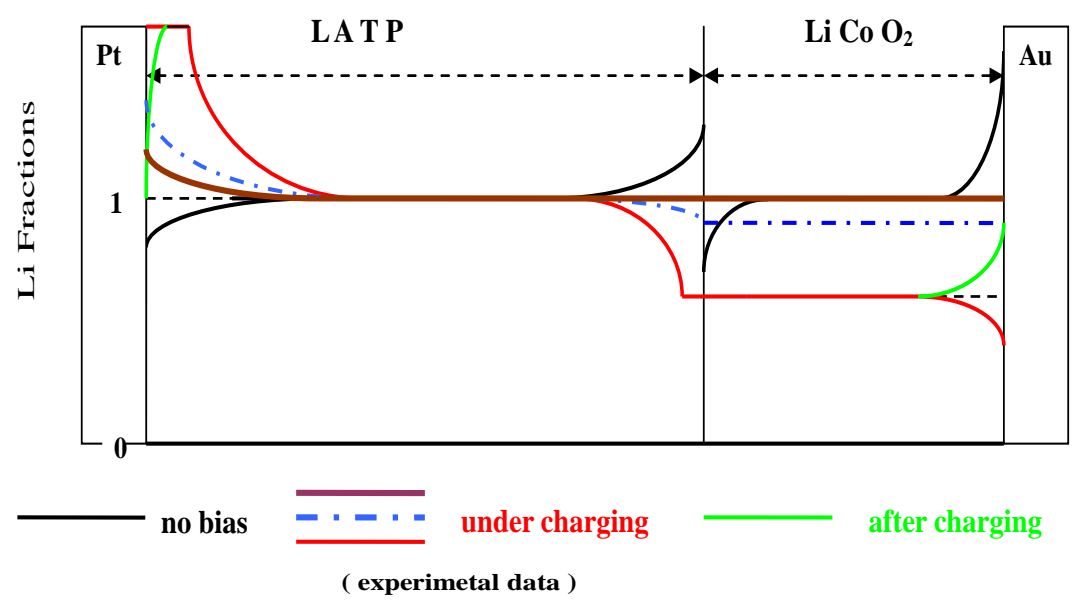

Figure 19 Schematic representation of the Li depth profiles in Au/LCO/LATP/Pt under conditions of charging, drawn using data presented in Figure 14 and Figure 18 [11].

\subsubsection{Change in Li Depth Profiles in LCO under Discharging Measured from Au Surface}

To determine the discharging characteristic of the Au/LCO/LATP/Pt battery from the Au surface (based on the result in Figure 19), at first, Au at $2.4 \mathrm{~V}$ was charged under conditions of bias for 25 min with respect to Pt at earth potential. Hereafter, it was discharged under biasing conditions at$0.5 \mathrm{~V}$ for $25 \mathrm{~min}$ and at $-1.0 \mathrm{~V},-1.5 \mathrm{~V}$, and $-2.2 \mathrm{~V}$ for $50 \mathrm{~min}$. At each stage, the ERD and RBS energy spectra were recorded simultaneously. The ERD spectra were normalized using the as-received one (at 0 V) [10] (Figure 20). The interfaces of the Au/LCO and LCO/LATP are indicated by dotted lines in the figure.

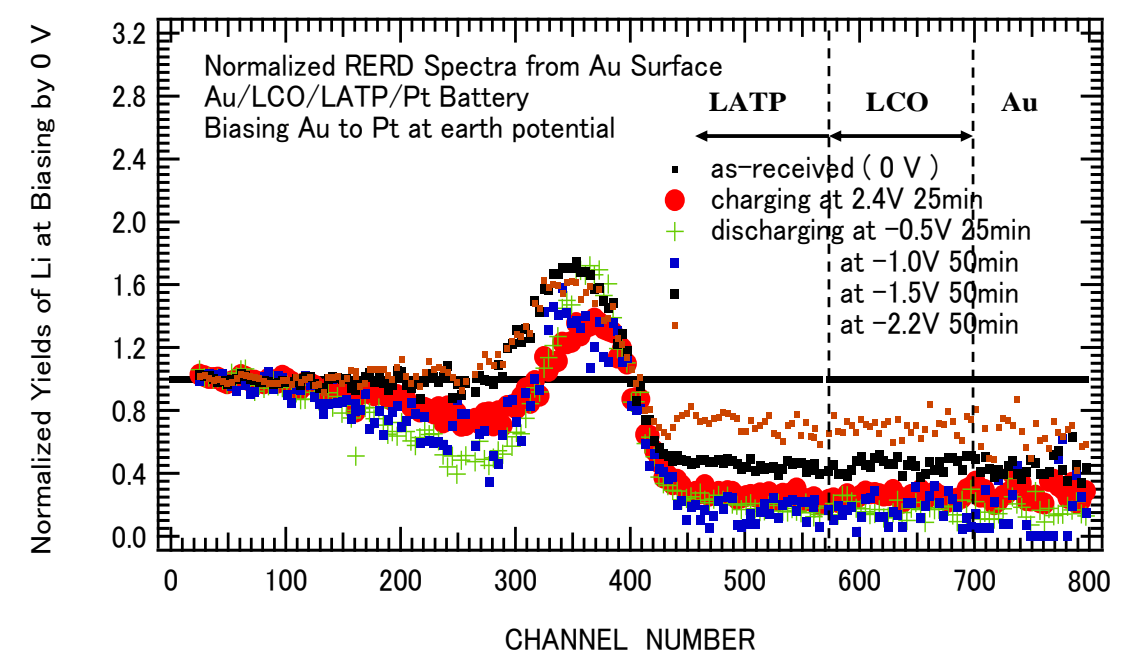

Figure 20 Normalized yields of Li measured under charging conditions at $2.4 \mathrm{~V}$ (time: 25 $\min )$. Following this, the system was discharged [11].

One can see from Figure 20 that the normalized ERD spectral profiles are very similar to the spectral profiles shown in Figure 14. It was also observed that $\mathrm{Li}$ in LCO is steadily reduced to $\sim 0.3$ under the initial charging conditions. Whereas, even under discharging conditions at $-0.5 \mathrm{~V}$ (time: $25 \mathrm{~min}$ ), Li did not get reduced. However, it was enriched under the next biasing conditions at -1.0 
$\mathrm{V}$ (time: $50 \mathrm{~min}$ ). The amount was recovered in the level on biasing at $1.5 \mathrm{~V}$ for $50 \mathrm{~min}$. Subsequently, it increased at $-2.2 \mathrm{~V}$ (time: $50 \mathrm{~min}$ ). The results indicated that the battery was reversible under charging and discharging conditions.

Such an anomalous change in Li in LCO is schematically shown in Figure 21, where the vertical axis represents the number of $\mathrm{Li}$ in LCO moved under biasing conditions (under conditions of charging and discharging, starting from $\mathbf{O}$ ). It was estimated by studying the Li composition (0.25) of the as-received LCO. The anomalous change observed and the normal change expected ( $\left.\mathbf{A}^{\prime}-\mathbf{D}\right)$ are indicated by thick solid $\left(\mathbf{A}^{\prime}-\mathbf{B}\right)$ and thin solid $(\mathbf{B}-\mathbf{C}-\mathbf{D})$ lines, respectively. The $\left(\mathbf{A}-\mathbf{A}^{\prime}\right)$ dasheddot-dashed-line represents the change in polarity upon biasing.

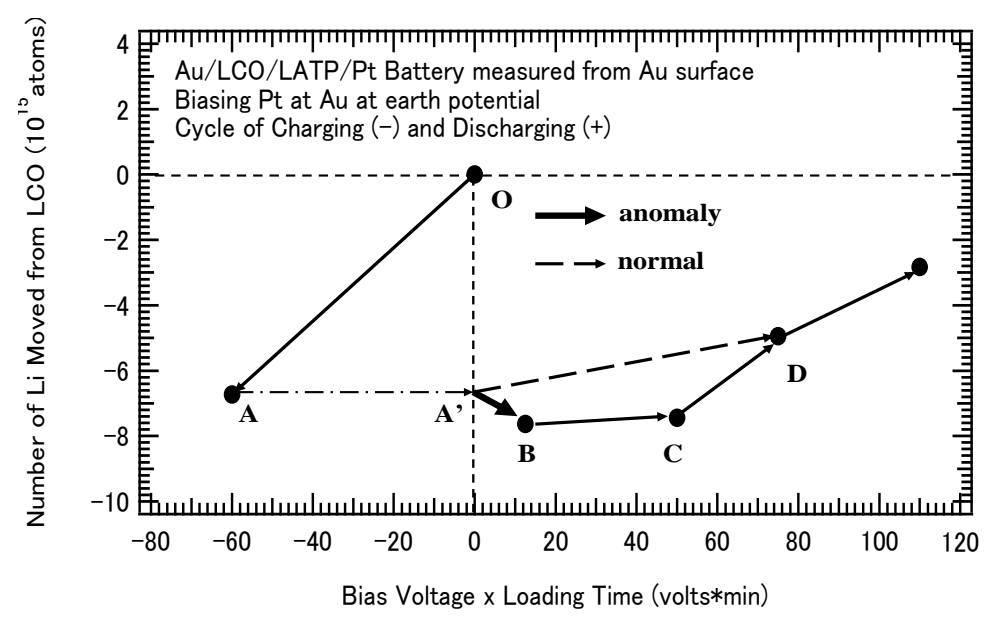

Figure $\mathbf{2 1} \mathrm{A}$ schematic representation of the bias-induced anomalous change in Li in LCO under charging and discharging conditions [11].

One can see from Figure 21 that the anomaly lies in the reduction in the number of Li under conditions of positive biasing, although it is expected to be enriched under discharging conditions. The anomaly can be potentially attributed to the over-reduction of Li in LCO (from 1 to 0.3; Figure 20). The reduction is induced during the first charging conditions (over-charging). The overreduction of $\mathrm{Li}$ resulted in the generation of the barrier at the interface, which prohibited the movement of Li from LCO to LATP. The barrier height was deduced to be $\sim 1.0 \mathrm{~V}$. The origin of the barrier can potentially be attributed to the change in the structure of LCO. This can also be attributed to the significantly large reduction in Li content. The interfacial interaction of Li with the hydrogen involved during its deposition might also explain this phenomenon, although no change in the Li composition was observed at the interface. It is noted that the anomaly observed is ascribed to the simple over-biasing under conditions of charging. Thus, a real battery can get damaged due to over-charging.

\subsubsection{Changes in the Li Depth Profiles Near Pt under Discharging: Measured from Pt Surface}

The changes in the Li depth profiles under discharging conditions (measured from the Pt surface) have been described [10]. At first, the system was charged during Pt biasing at $-2.4 \mathrm{~V}$ for $20 \mathrm{~min}$ (Au at earth potential was considered during the measurements). The results from the previous experiments revealed that the anomaly was observed under discharging conditions. Hence, the system was continuously discharged under biasing conditions at $1.0 \mathrm{~V}$ (time: $20 \mathrm{~min}$ and $40 \mathrm{~min}$ ). 
The normalized yields of $\mathrm{Li}$ at $\mathrm{OV}$ were obtained during the process (Figure 22). The thick solid line at unity represents the Li composition of the as-received specimen $(0 \mathrm{~V})$ and the interface of LATP/Pt is indicated by dashed lines.

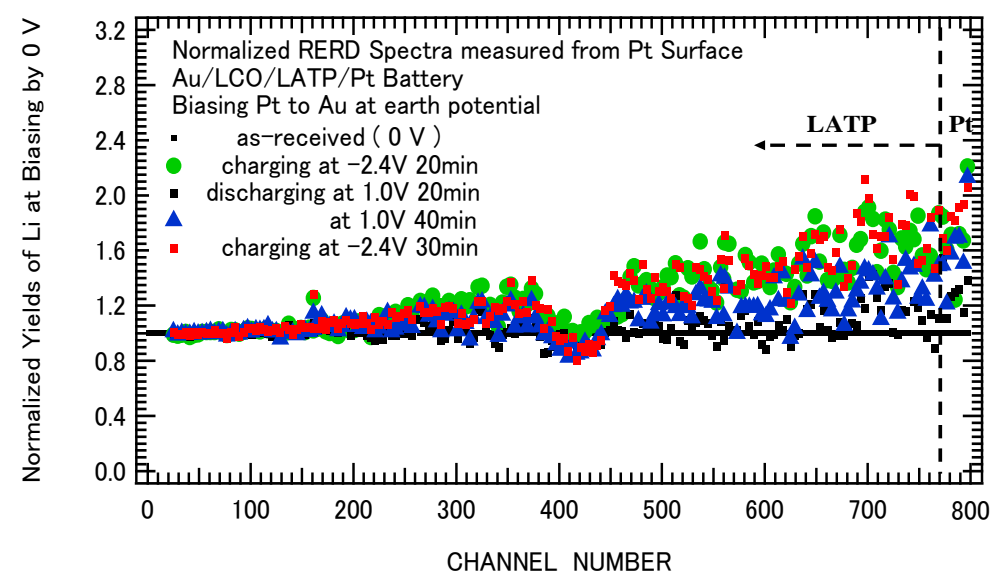

Figure 22 Normalized yields of Li by $0 \mathrm{~V}$ measured during the cycle of charging, discharging and charging from Pt surface [11].

One can see from Figure 22 that the Li yields ( $\bullet$ ) enriched by biasing at $-2.4 \mathrm{~V}$ for 20 min are considerably lower than those shown in Figure 18; thus, it is deduced that the extent of depletion of $\mathrm{Li}$ in LCO during charging is considerably lower than that presented in Figure 20. The value was found to be in the range of $0.3-0.4$. It was also observed that the Li yields significantly decrease to the values represented by $(\mathbf{a})$ during the process of discharging at $1.0 \mathrm{~V}$ (time: $20 \mathrm{~min}$ ). It is also seen that during an additional discharging process (time: $20 \mathrm{~min}$ ), an anomaly takes place for the Li yields. The Li yields increase to the level indicated by $\boldsymbol{\Delta}$. This also indicates that Li returns from LCO of the positive electrode to the negative electrode formed in LATP near the interface in the presence of Pt. However, such a virtual phenomenon does not take place in a real battery because the discharging process occurs in the absence of bias conditions. Only a connection with its load is maintained. It is also seen that during the final biasing at $-2.4 \mathrm{~V}$ (time: $30 \mathrm{~min}$ ), the Li yields ( $\square$ ) come back to the as-received level. The changes in the Li amounts presented in Figure 22 were also plotted in Figure 23. The method of plotting was similar to the method used for plotting Figure 21. The number of Li units in LATP during the charging process was estimated using the Li composition (0.154) of the as-received LATP. The anomalies are shown by thick solid lines. 


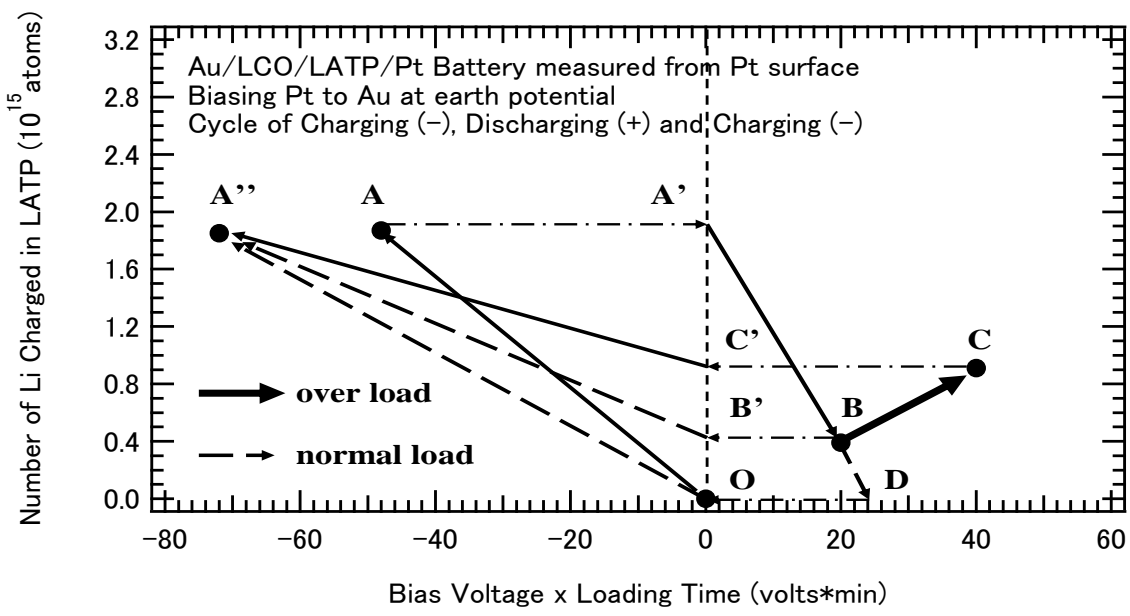

Figure 23 A schematic representation of the anomalous changes observed in Li in LATP induced by biasing under conditions of discharging [11].

One can see clearly from Figure 23 that the number of Li charged in LATP from LCO from $\mathbf{O}$ to $\mathbf{A}$ changes to $\mathbf{B}$ through $\mathbf{A}^{\prime}$ and then to $\mathbf{C}$ under discharging conditions. The number comes back to $\mathbf{A}^{\prime \prime}$ through $\mathbf{B}^{\prime}$ due to recharging. The increase of $\mathbf{L i}$ from $\mathbf{B}$ to $\mathbf{C}$ due to discharging is a strong anomaly that can be attributed to the difference in the prediction (from $\mathbf{B}$ to $\mathbf{D}$ to $\mathbf{A}$ through $\mathbf{O}$ ). It is also clear that when Li has been discharged from the negative electrode in the real battery to $\mathbf{B}$ (or $\mathbf{D}$ ), the recharging process under conditions of biasing is performed through B' (or $\mathbf{O}$ ) to A (or A"). Moreover, the amount of Li charged into LATP (Figure 23) is considerably smaller than that of Li reduced in LCO (Figure 21). The difference might be attributed to the loading times under charging conditions because the normalized $\mathrm{Li}$ yields presented in Figure 22 are considerably lower than those presented in Figure 14. The yields were measured from the Au surface under charging conditions that were similar to the conditions under which the experiment represented in Figure 20 was conducted.

The anomaly observed on additional biasing at $1.0 \mathrm{~V}$ (time: $20 \mathrm{~min}$; over-biasing) is reasonably explained in terms of the counter flow of the Li atoms in LATP by the biasing. It originates in the transport fraction of the $\mathrm{Li}$ ions (less than unity). When additional biasing under the discharging condition is applied at $\mathbf{B}$, the amounts of Li ions transported to LCO from LATP is once reduced down to zero. Nevertheless, the flow of the Li ions to LCO and the counter flow of the Li atoms to Pt are being formed be the potential gradient formed in LATP by the bias. But the Li ions transported to LCO have been nothing more in LATP. Thus, the counter flow of the Li atoms brings about the return of Li from LCO to LATP near the interface with Pt (B-D-C; Figure 23).

Finally, it is again emphasized that the battery fabricated using Au/LCO/LATP/Pt is rechargeable, and the anomaly is ascribed to the virtual over-load due to biasing.

\subsection{Ti/NbO/LiPON/LMO/Ti/Si (substrate) Battery under Conditions of Charging and Discharging}

\subsubsection{Change in the Li Depth Profiles in NbO under Charging Conditions}

In the Au/LCO/LATP/Pt battery described in the preceding section, the storage capacity of Li in the LATP near Pt electrode is significantly higher than the charging capacity of Li in LCO. The overreduction of Li from LCO to LATP was detected even under biasing conditions at $0.5 \mathrm{~V}$ during the 
discharging process. Li did not return to LCO. The changes in the Li depth profiles in the NbO film of the Ti/NbO/LiPON/LMO/Ti battery [12], where its storage capacity was limited by its thickness, were determined from the Ti/NbO side under charging conditions at several stages of biasing. The ERD energy spectra for the as-prepared specimen biased at -1.5, -2.5, 3.5, -4.5, and -5.5 $\vee$ for $15 \mathrm{~min}$ were recorded.

To closely inspect the changes in the ERD energy spectra under charging conditions, the spectra normalized by the one at $-4.5 \mathrm{~V}$, corresponding to the saturation of Li charged in the $\mathrm{NbO}$ film, were recorded (Figure 24 and Figure 25, respectively). The solid line at unity represents the saturation fraction of $\mathrm{Li}$ and the vertical solid line at 980 channels, dashed lines at 745 channels and 380 channels represent the Ti surface and interfaces of Ti/NbO and NbO/LiPON. The estimations were done using the thicknesses of the $\mathrm{Ti}$ and $\mathrm{NbO}$ films.

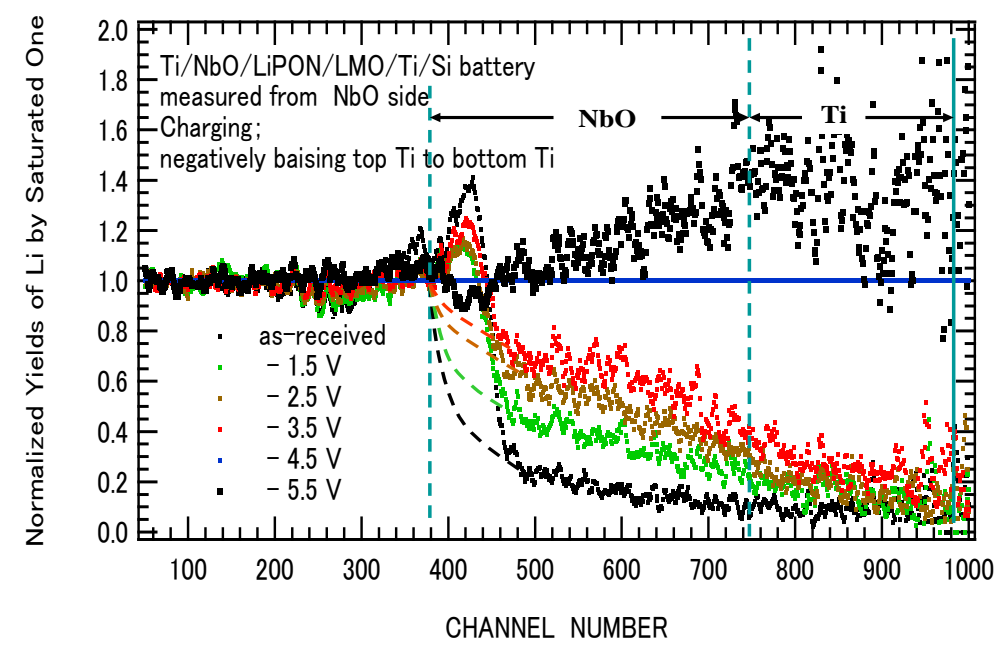

Figure 24 Normalized ERD spectra of Li charged in NbO under biasing conditions at -1.5, $-2.5,-3.5,-4.5$, and $-5.5 \vee$ compared to the one biased at $-4.5 \vee[12]$.

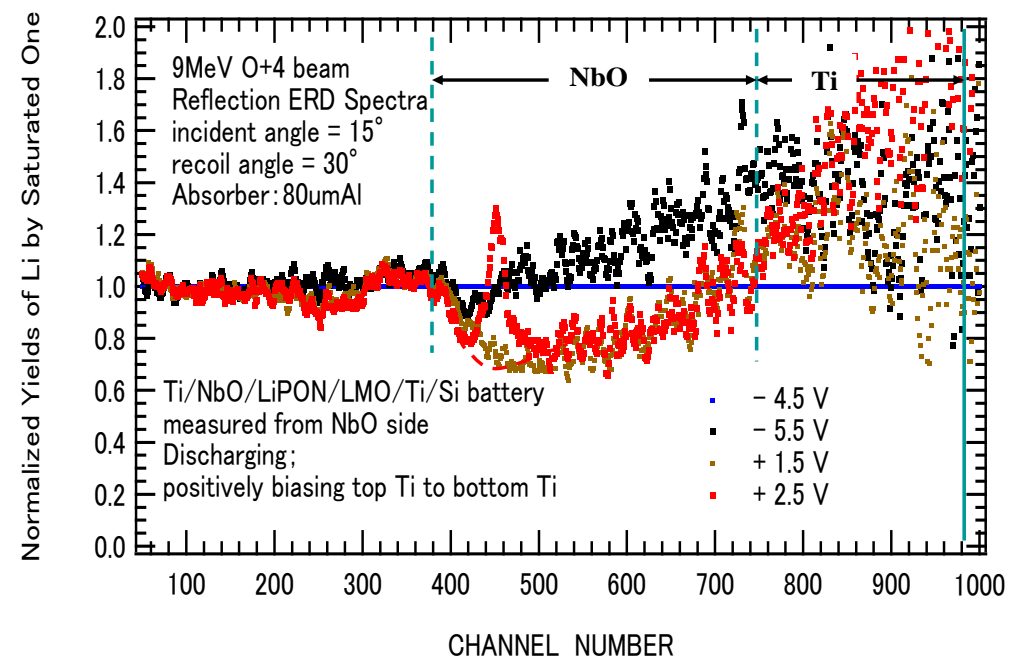

Figure 25 Normalized ERD spectra of Li discharge from $\mathrm{NbO}$ under biasing conditions at 1.5 and $2.5 \mathrm{~V}$ compared to the one biased at $-4.5 \mathrm{~V}$ [12]. 
It is seen from Figure 24 that the Li composition in $\mathrm{NbO}$ increases with decreasing bias voltage. Saturation is attained due to the decrease in the bias voltage from $-3.5 \mathrm{~V}$ to $-4.5 \mathrm{~V}$. The increments exhibit diffusing slopes in the direction from LiPON to the Ti electrode. Moreover, it is seen that biasing at $-5.5 \mathrm{~V}$ results in the diffusion of $\mathrm{Li}$ into the Ti electrode, although the absolute amounts of $\mathrm{Li}$ are not significantly large. The height of the sharp peak at 420 channel decreases with a decrease in the bias voltage. This can be attributed to the desorption of $\mathrm{H}$ in the top Ti electrode. On the other hand, it is also seen from Figure 25 that $\mathrm{Li}$ once charged in $\mathrm{NbO}$ and diffusing into the Ti electrode is reduced during the subsequent discharging processes occurring at 1.5 and $2.5 \mathrm{~V}$. The decrements exhibit diffusing slopes in the direction opposite to charging. These results indicate that the diffusion of $\mathrm{Li}$ in $\mathrm{NbO}$ is slower than that in LCO (described in the preceding section). Moreover, an analysis of the ERD spectrum revealed that a sharp peak corresponding to $\mathrm{H}$ at the surface of the top $\mathrm{Ti}$ electrode grows due to biasing at $2.5 \mathrm{~V}$. The growth of $\mathrm{H}$ can be potentially ascribed to the reaction between the Li segregated at the Ti surface and the residual $\mathrm{H}_{2} \mathrm{O}$ vapor in vacuum (as the Li yields near the 980 channels in Figure 25 are enhanced).

The composition of $\mathrm{Li}$ charged in the $\mathrm{NbO}$ film up to the point of saturation can be estimated using the RBS energy spectra measured simultaneously with the ERD spectra, which are shown in Figure 26. It is seen from Figure 26 that the RBS yields from Nb around 750 channels at -4.5 and $5.5 \mathrm{~V}$ are lower than the yields obtained from the as-prepared one, which originates during the charging of $\mathrm{Li}$ into $\mathrm{NbO}$ (Figure 24). As $\mathrm{H}$ is also involved in the $\mathrm{NbO}$ film, the $\mathrm{Li}$ and $\mathrm{H}$ compositions should be determined simultaneously. The ratio of the $\mathrm{Li}$ and $\mathrm{H}$ compositions can be determined from the ERD spectra, and the values of the $\mathrm{Li}$ and $\mathrm{H}$ contents can be determined from the extent of the reduction in the height of the peaks representing the RBS yields and the composition ratio. The composition of the $\mathrm{NbO}$ film at saturation under charging has been estimated to be $\mathrm{Li}_{2.6} \mathrm{H}_{0.7} \mathrm{Nb}_{2} \mathrm{O}_{5}$. The $\mathrm{Li}$ composition in the as-prepared sample has been roughly estimated to be $\mathrm{Li}_{0.52} \mathrm{H}_{0.7} \mathrm{Nb}_{2} \mathrm{O}_{5}$ on the assumption that the $\mathrm{H}$ composition does not change due to charging.

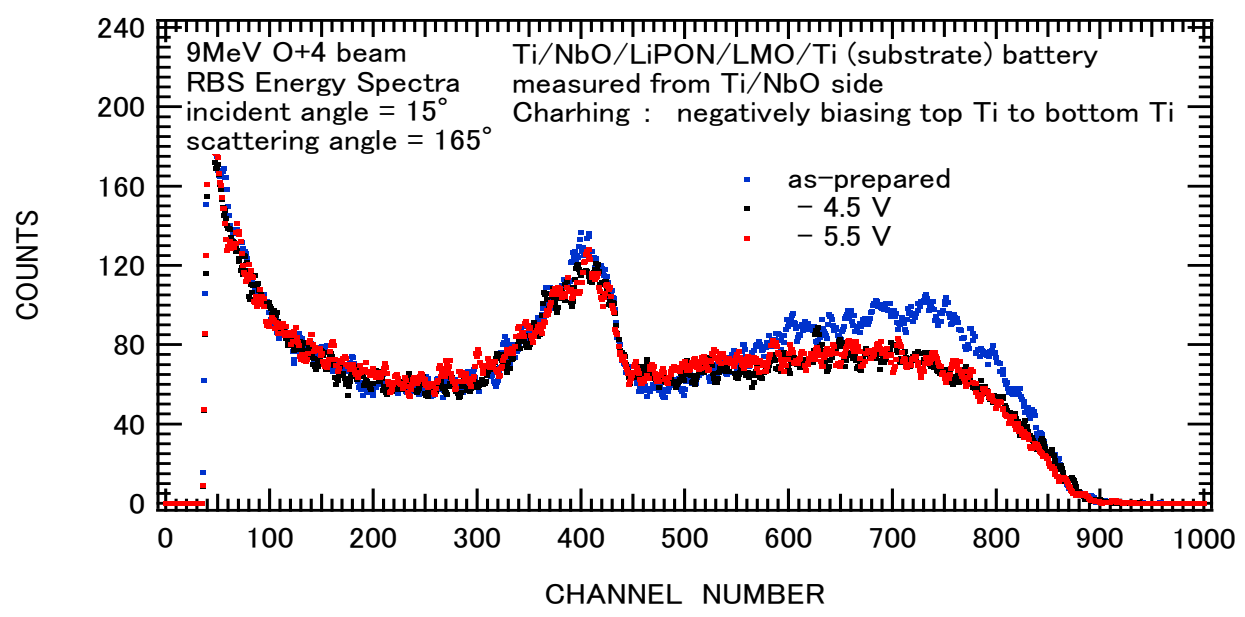

Figure 26 RBS energy spectra from the specimen as-received, at -4.5 and $-5.5 \mathrm{~V}$ measured simultaneously with ERD [12].

The variation in the Li composition in the $\mathrm{NbO}$ film under biasing conditions on charging and discharging obtained from Figure 24 and Figure 25 is summarized in Figure 27, where the bias voltage was increased along the solid arrows and the dash-dot-dash arrows represent the inverse 
of the polarity of the bias voltage. One can see from the figure that upon charging, the Li content in $\mathrm{NbO}$ increases from 0.52 to 2.6 when the bias voltage decreases. The charging amount of Li content is 2.08. Upon discharging, the decrease in the amount of $L i$ is only 0.5 . The result indicates that the battery is rechargeable, but the difference between the amounts of Li transported under conditions of charging and discharging is significantly large, and the efficiency in its cycle performance is significantly low. The results agree well with the data presented in the literature [16]. The origin for $\mathrm{Li}$ (under charging conditions) and its property to remain in $\mathrm{NbO}$ can potentially be ascribed to the formation of stable Li compounds such as $\mathrm{LiOH}$ due to the involvement of $\mathrm{H}$ in $\mathrm{NbO}$.

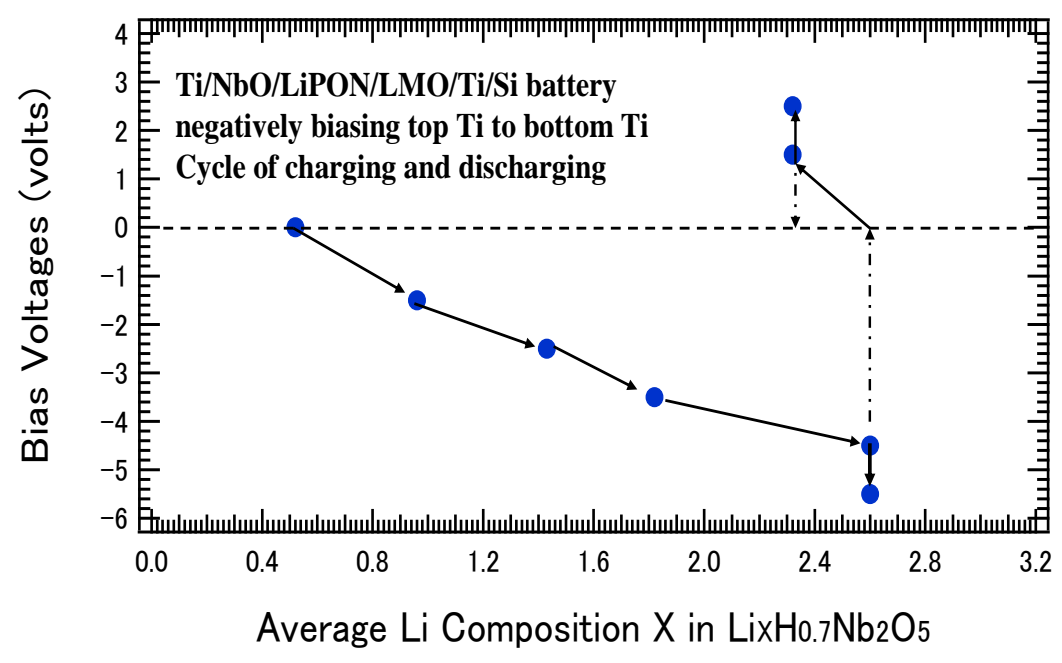

Figure $\mathbf{2 7}$ The relation of Li composition in $\mathrm{NbO}$ with the bias voltage under charging and discharging conditions [12].

\subsubsection{Change in the Li Depth Profiles in LMO under Discharging Conditions}

Here, the changes in the Li depth profiles in the LMO film of the Ti/LMO/LiPON/NbO/Ti/Si battery [12] are presented in detail. The measurements were taken from the Ti/LMO side at several stages of biasing during discharging and charging cycles. The ERD energy spectra were recorded at bias voltages of $0,-2.0,-2.5,-3.0$, and $-3.5 \mathrm{~V}$ on discharging and subsequently at bias voltages of $2.0,3.0$, 4.0 , and $4.5 \mathrm{~V}$ on charging.

To closely inspect the bias-induced changes in the Li composition in the LMO film, the ERD spectra were normalized by the one at $-3.5 \mathrm{~V}$. The result indicates that the Li composition was saturated to produce $\mathrm{LiMn}_{2} \mathrm{O}_{4}$ under discharging conditions. The normalized ERD spectra under discharging and charging conditions are shown in Figure 28 and Figure 29, where the energies of $\mathrm{Li}$ and $\mathrm{H}$ recoiled at the Ti surface. The interfaces of LMO/Ti and LiPON/LMO were determined considering the thicknesses of LMO and Ti. The dotted lines show the changes in the spectral profiles. 


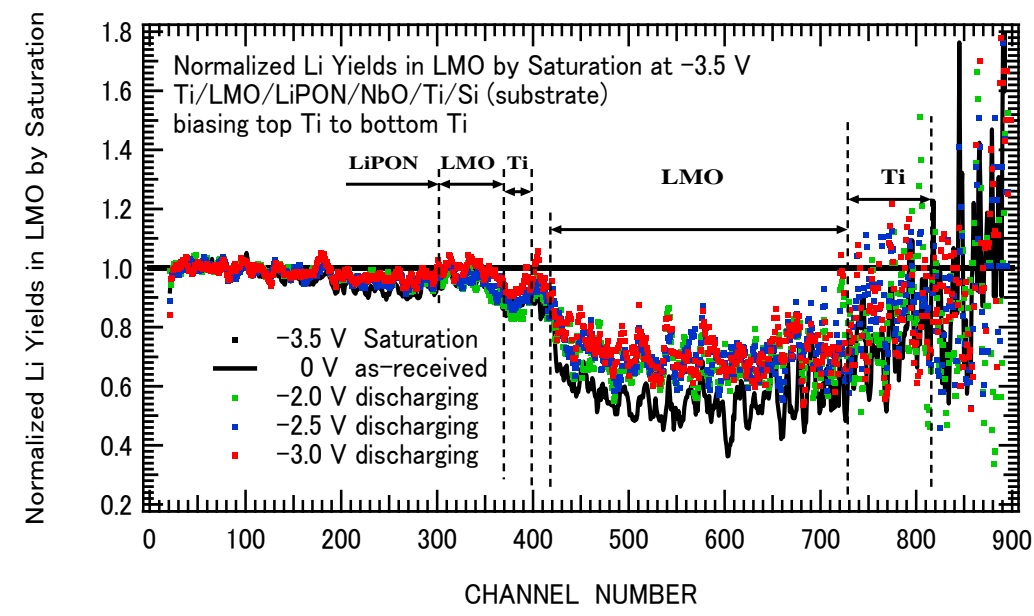

Figure 28 ERD spectral profiles recorded at $0,-2,-2.5$, and $-3 \vee$ normalized by saturation at $-3.5 \mathrm{~V}$. The Ti surface and the interfaces of Ti/LMO and LMO/LiPON are shown by dotted lines [12].

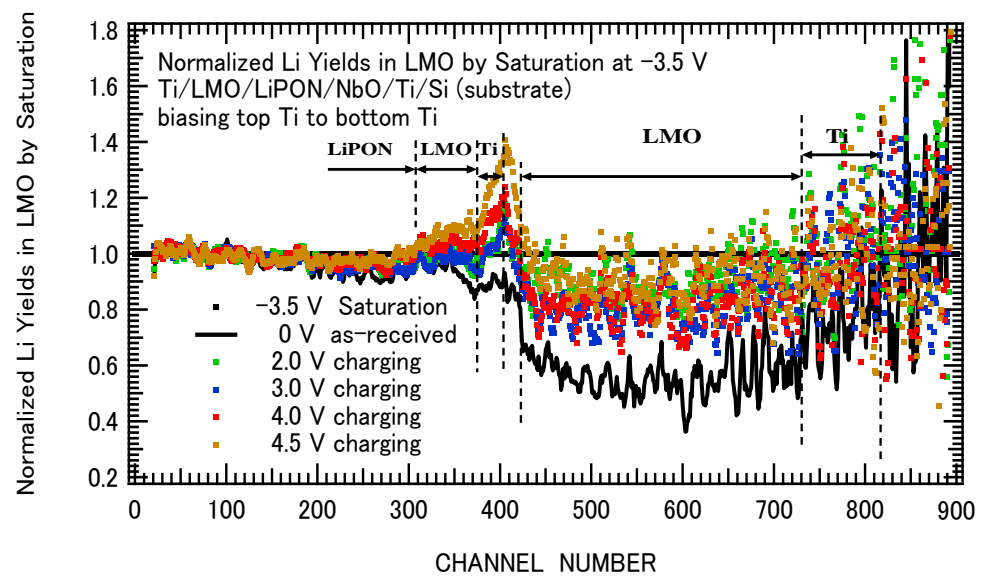

Figure 29 ERD spectral profiles recorded at 0, 2, 3, 4, and $4.5 \mathrm{~V}$ normalized by saturation at $-3.5 \mathrm{~V}$. The Ti surface and the interfaces of Ti/LMO and LMO/LiPON are shown by dotted lines [12].

It is seen from Figure 28 and Figure 29 that the normalized yields of $\mathrm{Li}$ in LMO increase with increasing bias voltage upon discharging and its distribution is uniform over the whole depth of LMO. This indicates that an ordinary discharge process is followed. The uniform distribution of $\mathrm{Li}$ in LMO is very similar to that in LCO described in the Au/LCO/LATP/Pt battery [9]. It is also seen from Figure 28 that the Ti yields increase due to the biasing. Figure 29 reveals that upon subsequent charging, the $\mathrm{Li}$ yields in LMO at first decrease and then increase with increasing bias voltage. Furthermore, the peak yields of $\mathrm{H}$ around 400 channels corresponding to the Ti surface increase steeply at 4.0 and $4.5 \mathrm{~V}$. This can be attributed to the chemical reaction between the Li segregated at the Ti surface and residual $\mathrm{H}_{2} \mathrm{O}$ vapor in the vacuum. The event is described below.

The variation in the Li composition in LMO with the bias voltage in one cycle of discharging and charging is summarized in Figure 30, where the dot-dash-dot line represents the switch-off of the polarity of bias voltage. It is seen from Figure 30 that during the initial discharging phase, the Li composition decreases (from 0.55 ) and reaches a saturated value. The value decreases as the bias voltage decreases. On subsequent charging, it decreases with increasing voltage but again increases 
to 4.0 and $4.5 \mathrm{~V}$. The increase in the Li composition in LMO during the charging process indicates that an anomaly takes place in the Li transport in the battery. The anomaly is reasonably explained in terms of the $\mathrm{Li}$ composition in $\mathrm{NbO}$. The amounts of $\mathrm{Li}$ are saturated by charging till 3.0 V. But $\mathrm{Li}$ over-saturated in $\mathrm{NbO}$ at $4.0 \mathrm{~V}$ does not diffuse into the Ti and Si substrates in the follow direction and returns to LMO via the diffusion of the Li atoms in the counter direction in the LiPON electrolyte because the transport fraction of the Li ions in the electrolyte is below unity. It is similar to the anomaly observed upon overcharging the Au/LCO/LATP/Pt battery described in the previous section. Moreover, the Li atoms returning to LMO seem to diffuse into $\mathrm{Ti}$ and segregate at the surface (Figure 29). This is consistent with the increase in the $\mathrm{H}$ yields at 4.0 and $4.5 \mathrm{~V}$, which is also observed in Figure 31. The return of Li to LMO is attributed to the phenomena of repelling the Li-ions under conditions of the positive potential barrier. This is generated by the difference in the Fermi energies at the interface between $\mathrm{NbO}$ and $\mathrm{Ti}$ on the Si substrate, namely, the Schottky barrier. The result contradicts the previous result, whereupon over-charging the Ti/NbO/LiPON/LMO/Ti/Si substrate battery resulted in the overflow of $\mathrm{Li}$ in the $\mathrm{NbO}$. It subsequently diffuses into the Ti film (Section 41).

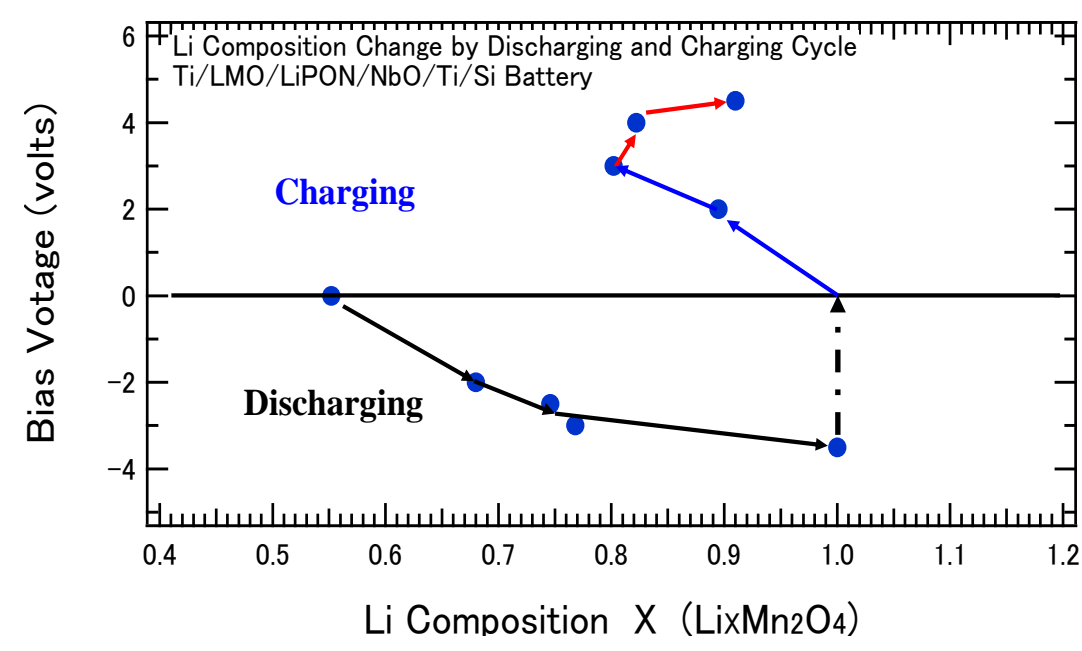

Figure 30 Variation in the Li composition in LMO under the influence of the bias voltages under conditions of discharging and charging. The dot-dash-dot line represents the switch-off of the polarity of the bias voltage [12].

It is also seen from Figure 30 that Li in LMO is depleted by only 0.2 (decreases from 1.0 to 0.8 ). This decrease is also ascribed to the formation of stable Li compounds such as LiOH due to the involvement of $\mathrm{H}$ present in the as-prepared LMO. The results are similar to the results obtained by studying the system from the Ti/NbO side (described above). As the amounts of Li moving under conditions of charging and discharging indicate the efficiency of a real battery, further systematic studies on the $\mathrm{H}$-free battery need to be conducted. 


\section{Results of the Studies on Li Depth Profiling of Thin Film-Stacked Micro-Li Ion Batteries Conducted using the TERD Technique}

In this section, the total Li depth profiles of the thin film-stacked micro-batteries, which were determined under conditions of biasing using the TERD technique with the newly developed 5MeV $\mathrm{He}^{+2}$ ions, have been discussed $[7,8]$.

\subsection{Charging and Discharging Characteristics of the LCO/LiPON/Si Batteries}

Here, the results obtained upon charging the $\mathrm{Au} / \mathrm{LCO} / \mathrm{LiPON} / \mathrm{Si} / \mathrm{Au} / \mathrm{Al}$ and $\mathrm{Ni} / \mathrm{LCO} / \mathrm{LiPON} / \mathrm{Si} / \mathrm{Pt} / \mathrm{Ti}$ batteries [8] have been presented. The RBS and TERD spectra of the $5.4 \mathrm{MeVHe}^{+2}$ ions from the $\mathrm{Al} / \mathrm{Au} / \mathrm{LCO} / \mathrm{LiPON} / \mathrm{Si} / \mathrm{Au}$ battery biased at $0,+1.0$, and $+1.3 \mathrm{~V}$ are shown in Figure 32 and Figure 33, respectively. In Figure 32, the energy positions of the He units backscattered from the surface of the Al substrate, top Au film, Co, Si, and bottom Au film are marked by arrows. In Figure 33, the energy positions of the He units backscattered from $\mathrm{Li}$ and $\mathrm{Li}$ recoil brought about by incident $\mathrm{He}$ from the interfaces of LCO, LiPON, and $\mathrm{Si}$ are also marked by arrows. The thickness of each film is also shown. The background yields except for Li recoils below 400 channels are attributed to the recoils of the higher mass constituent elements such $\mathrm{N}, \mathrm{O}, \mathrm{Si}$, and $\mathrm{P}$ in the specimen. The background yields are rather high, which is ascribed to multiple scattering attributable to the high thickness of LiPON. It is expected that they can be easily reduced by inserting $2 \mu \mathrm{m}$-thick Al absorber film. The energy resolution is slightly reduced.

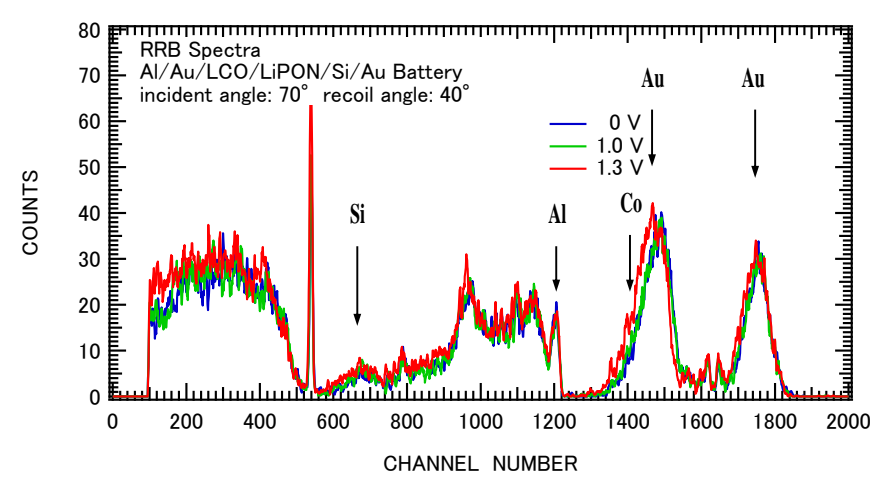

Figure 31 RBS spectra of the 5.4 $\mathrm{MeV} \mathrm{He}^{+2}$ scattered from Al/Au/LCO/LiPON/Si/Au [7].

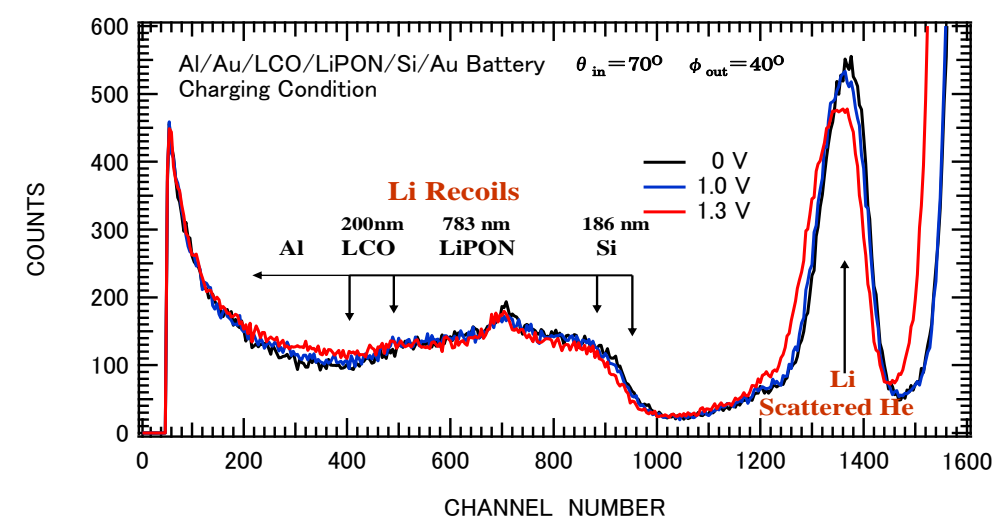

Figure 32 TERD energy spectra of 5.4 MeV-He $\mathrm{Me}^{+2}$ scattered from Al/Au/LCO/LiPON/Si/Au [7]. 


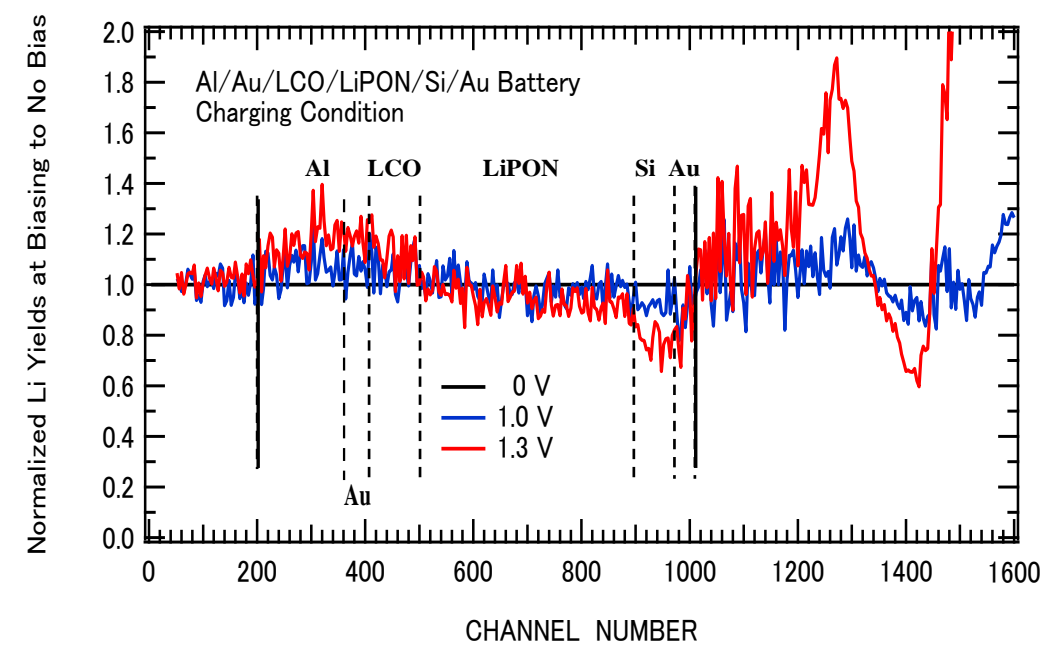

Figure 33 Normalized TERD spectra at 1.0 and $1.3 \mathrm{~V}$ to $0 \mathrm{~V}$ [7].

It is noted in Figure 32 that the energy of the Au peak from the bottom film at $1.3 \mathrm{~V}$ shifts toward the region of lower energy. This contradicts the results observed by analyzing the RBS spectra shown in Figure 31, which reveals that the total stopping power of LCO + LiPON + Si between both the Au films increases due to a rise in the bias voltage (from 2.0 to $2.8 \mathrm{~V}$ ). This point is discussed below, along with the results obtained by analyzing the TERD spectral profiles. To understand the Li movement under biasing conditions, the TERD spectral profiles at 1.0 and $1.3 \mathrm{~V}$ normalized to $0 \mathrm{~V}$ (obtained from Figure 32) are shown (Figure 34). Here, the positions of each interface between the constituent films and the top and bottom Au surfaces are shown by dashed and solid lines, respectively. The peak widths of the corresponding Au peaks presented in Figure 32 were analyzed to obtain the results.

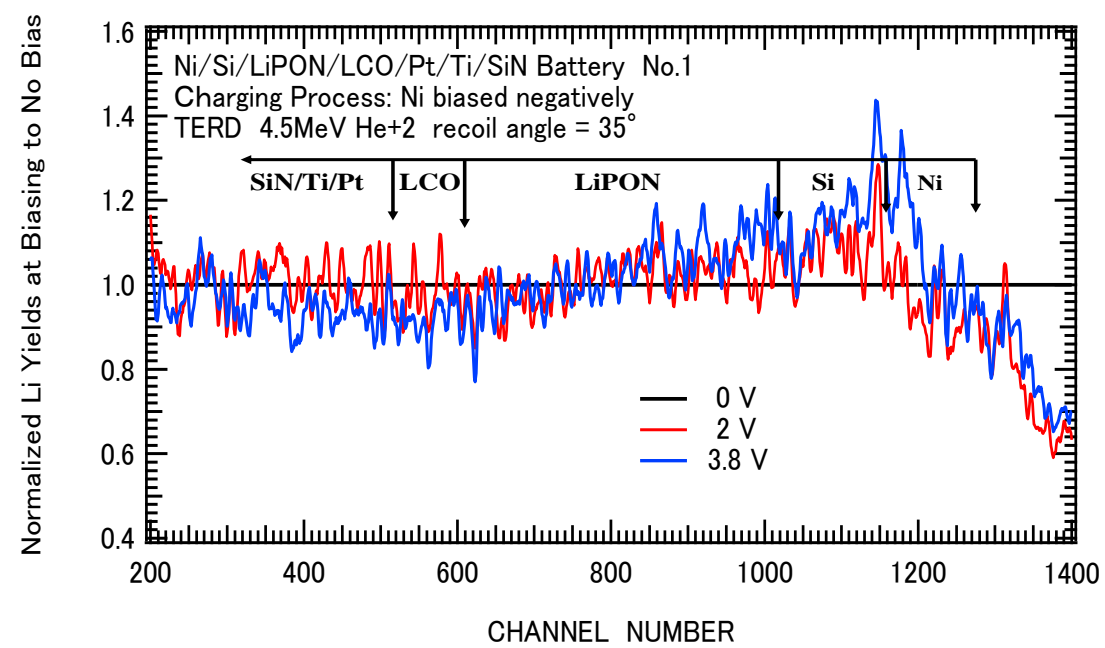

Figure 34 TERD spectral profiles recorded for Ni/Si/LiPON/LCO/Pt/Ti/SiN biased at 2.0 and $3.8 \mathrm{~V}$ and normalized by $0 \mathrm{~V}[8]$.

One can see from the TERD spectral profiles presented in Figure 34 that the Li yields obtained from the Si film decrease. On the contrary, the yields from the LCO and Al films increase with increasing bias voltage even under conditions of charging. The decrease in the Li yields from Si due to biasing is more clearly observed in the normalized TERD spectral profiles presented in Figure 32, 
where the change in the Li yields from each constituent film are differentiated. This is possible because of the high depth resolution at the interfaces (marked by dashed lines). This result indicates that a large amount of Li in LCO has been transported to Si during the preparation of the specimen. The Li yields from the Si film at $0 \mathrm{~V}$ are significantly high. The opposite transport of Li under conditions of biasing can also be seen from the shift in the position of the large He peak scattered from Li between 1200 and 1450 channels (Figure 34). This result is similar to the results obtained with $9 \mathrm{MeV} \mathrm{O}^{+4}$ TERD [7], which shows that Li returns from Si into LCO and leaks into the Al substrate in the counter direction (against biasing). This can be attributed to the phenomenon of overcharging described previously. As the leakage of Li between the two Au films into the Al substrate should bring about a decrease in the stopping power of LCO + LiPON + Si, the increase in the stopping power between both the Au films observed in the RBS spectral profiles presented in Figure 32 indicates that the up-take of the $Z$ element in higher amounts (compared to Li). In such a case, it is assumed that Al might move in the counter direction (against biasing) through the Au film into LCO, LiPON, and Si. Thus, the leakage of Li into the Al substrate may be attributed to the mutual diffusion of the neutral $\mathrm{Li}$ and $\mathrm{Al}$ units through the Au film. A change in the Al film due to diffusion may not be observed in the RBS spectral profiles. The origin of Li leakage has been discussed in detail in the following section. The results obtained by studying Ni/Si/LiPON/LCO/Pt/Ti/SiN have also been presented. These results seem to indicate that a combination of the TERD and RBS techniques can be efficiently used to test the degradation of the battery performance under conditions of repeated charging and discharging. The changes in the Li depth profiles can be recorded to arrive at the results.

The results obtained by studying the two specimens fabricated using Ni/Si/LiPON/LCO/Pt/Ti/SiN have been presented. The constituent films were deposited simultaneously (except LCO, which was deposited separately). However, the amounts of Li taken up by Si via the process of diffusion under conditions of heating by magnetron plasma during the process of deposition were different. Firstly, the results obtained from the No.1 specimen, where Li was not fully up-taken, have been discussed. The RBS spectra and TERD spectra of the $4.5 \mathrm{MeV} \mathrm{He}+2$ ions from the Ni/Si/LiPON/LCO/Pt/Ti/SiN battery biased at $0,2.0$, and $3.8 \mathrm{~V}$ and the corresponding TERD spectra at 2.0 and $3.8 \mathrm{~V}$ were recorded simultaneously.

It has been found that the energy of the $\mathrm{Ni}$ peak at $3.8 \mathrm{~V}$ shifts toward the higher energy region (compared to those of $\mathrm{Pt}, \mathrm{Ti}, \mathrm{Co}$, and $\mathrm{Si}$ in $\mathrm{SiN}$, where the peaks overlap with each other). This result indicates that the stopping power of Si/LiPON/LCO was reduced when the bias voltage was increased from 0 and $2 \mathrm{~V}$ to $3.8 \mathrm{~V}$. This indicates the leakage of Li. Analysis of the TERD spectral profiles indicates that as the bias voltage increases, the Li yields from LCO decrease and those from Si increase. This indicates that the regular charging of Li takes place from LCO (cathode) to Si (anode). To inspect the changes in the Li yields over the whole battery, the TERD spectra recorded for Li at 2.0 and $3.8 \mathrm{~V}$ (normalized by $0 \mathrm{~V}$ ) are shown in Figure 35. The energies of the Li units recoiled from the Ni surface and at the interfaces of $\mathrm{Ni}, \mathrm{Si}$, LiPON, LCO, and Pt/Ti/SiN films are indicated by arrows. The horizontal straight line at unity corresponds to the Li yields at $0 \mathrm{~V}$. 


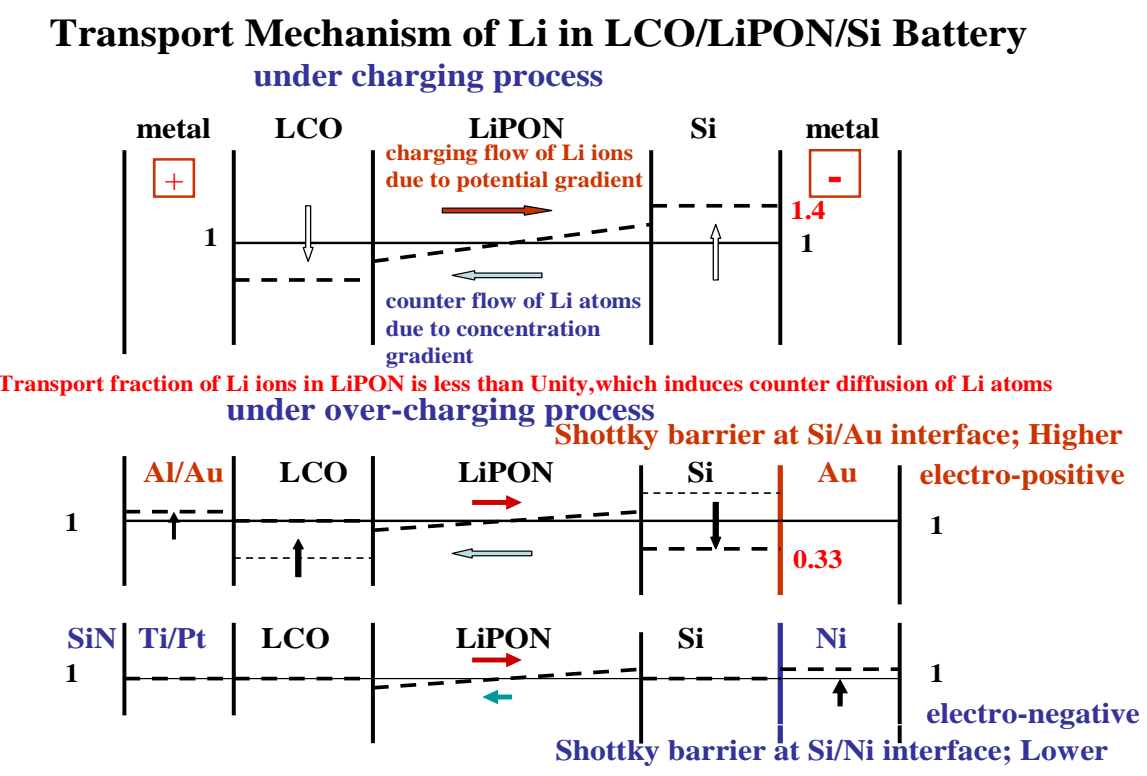

Figure 35 Schematic representation of the changes in the Li depth profiles of the LCO/LiPON/Si battery with Al/Au-Au and Ti/Pt-Ni metal electrodes at both sides under conditions of charging and over-charging [8].

It is seen from Figure 35 that the Li yields at 2.0 and 3.8 $\mathrm{V}$ from LCO and SiN/Ti/Pt films decreases (below unity) and those from Si and Ni near the interface increases above unity with increasing bias voltage. The former indicates the ordinary charging from LCO to Si through LiPON and the latter indicates that $\mathrm{Li}$ was up-taken in $\mathrm{Pt} / \mathrm{Ti} / \mathrm{SiN}$ during the preparation of the sample. Moreover, one can see that the Li yields from the LiPON film get depleted near LCO and enriched near Si. The yields in $\mathrm{SiN} / \mathrm{Ti} / \mathrm{Pt}$ are reduced under conditions of biasing. This corresponds well with the metal/LiPON/metal capacitor obtained using the conventional ERD technique in the presence of the $9 \mathrm{MeV} \mathrm{O}^{+4}$ ions [9]. The increase in the Li yields from Ni near Si at 3.8 $\mathrm{V}$ indicates the leakage of $\mathrm{Li}$ into the Ni film, as the decrease in the stopping power of Si/LiPON/LCO in the RBS spectra is ascribed to the reduction in the $\mathrm{Li}$ content. This fact may indicate that the biasing at $3.8 \mathrm{~V}$ results in full charging or over-charging (as described for the No.2 specimen below). The saturation composition of $\mathrm{Li}$ in $\mathrm{Si}$ was estimated to be $\mathrm{Li}_{1.4} \mathrm{Si}$. The reference was $\mathrm{Li}_{3.1} \mathrm{PO}_{3.8} \mathrm{~N}_{0.2}$.

In the following section, the results of studying the $\mathrm{No}_{2}$ specimen in $\mathrm{Si}$ of which $\mathrm{Li}$ was found to be almost fully up-taken have been described. The RBS and TERD spectra have been recorded at normal incidence and a recoil angle of $35^{\circ}$ for the specimen biased at $0,2.0$, and $3.8 \mathrm{~V}$. It has been found from the RBS spectra that the energy of the Ni peak at $3.8 \mathrm{~V}$ shifts toward the lower energy region against those at 0 and $2.0 \mathrm{~V}$, indicating that the stopping power of LCO/LiPON/Si increases under biasing conditions. It is essentially different from the previous result obtained by studying the No.1 specimen, where the stopping power decreases due to operations under similar biasing conditions. The differences in the stopping powers between the specimens and the TERD spectra are discussed below.

It has been observed in the ERD spectra that although the bias is applied under charging conditions, the Li yields from $\mathrm{Si}$ of the three TERD spectra are almost the same except that in the region of the Ni film. This indicates that the amounts of Li up-taken in Si of the as-prepared specimen 
were almost saturated. It has also been found from the ERD spectra that the Li yield from Si obtained by studying the height of the spectrum at $0 \mathrm{~V}$ is close to that at $3.8 \mathrm{~V}$.

The enrichment of Li over the whole Ni film under biasing conditions is attributed to the transport from the LCO and Pt/Ti/SiN films, as similar transport of Li was observed during the charging of the No.1 specimen. It has not been observed yet because of the high background yields. The increase in the stopping power of Si/LiPON/LCO in the RBS spectrum at $3.8 \mathrm{~V}$ is reasonably explained in terms of the flow-in of the high-Z element attributable to the mutual diffusion of Ni with Li at the interface of the Si and Ni films. This compensates for the decrease in the Li content because it brings about an increase in the stopping power. The Si-Ni interfacial reaction can potentially assist the diffusion of $\mathrm{Ni}$ and Li.

So far, it has been shown that upon over-charging, the leakage of Li from $\mathrm{Si}$ into $\mathrm{Ni}$ in the $\mathrm{Ni} / \mathrm{Si} / \mathrm{LiPON} / \mathrm{LCO} / \mathrm{Pt} / \mathrm{Ti} / \mathrm{SiN}$ battery in the following direction is significantly different from that observed for the Au/Si/LiPON/LCO/Au/Al battery, where the leakage of Li from Si into Al in the counter direction takes place. The experimental results obtained under conditions of charging and over-charging in Figure 34 and Figure 35 are summarized schematically in Figure 31, where the compositions of the Li saturated and reduced in Si under conditions of charging and over-charging, respectively, are also shown. Here, the difference is discussed in terms of the transport process of $\mathrm{Li}$ in LiPON under charging conditions. The differences in the Fermi energies of the pair of metal electrodes (Au-Au/Al and Ni-Pt/Ti) at both sides of Si/LiPON/LCO and that are in contact potentials of $\mathrm{Au}$ and $\mathrm{Ni}$ with $\mathrm{Si}$ have also been discussed. When a certain positive voltage is applied to the metal electrode in contact with the LCO (cathode), Li is transported from LCO through LiPON to Si (anode). The Li compositions in LiPON near LCO and Si are reduced and enriched, respectively, by reaching a balance in the quasi-equilibrium between the charging flow of Li ions due to the potential gradient and the counter flow of $\mathrm{Li}$ atoms due to the concentration gradient. This is because the transport fraction of the Li ions in LiPON under bias is less than unity, as described in the previous Section 3. When the system is overcharged when the amounts of Li charged in Si attain saturation, the potential gradient is maintained in LiPON under additional biasing conditions (to Au in contact with LCO). Although the extent of both charging follow and counter flow are reduced, the Li ions over-flowed out of $\mathrm{Si}$ is repelled by the Schottky barrier formed at the interface of Au/Si and they return to LCO because the counter flow of the Li atoms due to the concentration gradient is larger than the follow flow. It reacts with the more electro-negative Au/Al substrate, the Fermi energy of which is higher than that of Au. On the other hand, in the case of the Ni-Pt/Ti electrodes, as the follow flow is higher than the counter flow, the Li-ions over-flowed out of $\mathrm{Si}$ also react with the more electro-negative $\mathrm{Ni}$, the Fermi energy of which is higher than that of Pt/Ti. This can be attributed to the absence of a barrier or the lower barrier height of $\mathrm{Ni} / \mathrm{Si}$ than $\mathrm{Au} / \mathrm{Si}$ interfaces. This fact seems to indicate that $\mathrm{Ni}$ with the lower Fermi energy is preferred over the electrode in contact with Si (anode) than Au because the battery is not defective but available.

\subsection{Charging and Discharging Characteristics of the LMO/LiPON/NbO Batteries}

In this experiment, to reduce the background yields of the recoils with masses larger than Li, a $0.8 \mu \mathrm{m}$-thick Al absorber was inserted at the front of the solid-state detector. The process was similar to the process followed for studying the $\mathrm{O}$ ions. 
The changes in the Li depth profiles of the Ti/NbO/LiPON/LMO/Al substrate under discharging conditions are shown in Figure 36 [13].

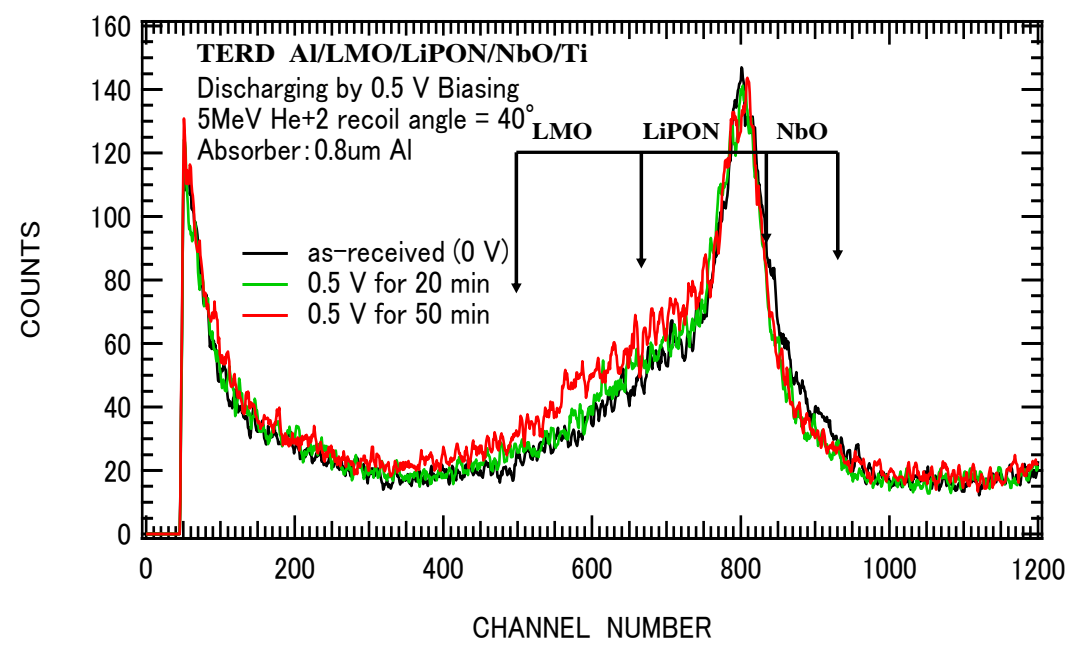

Figure 36 TERD spectral profiles of the Ti/NbO/LiPON/LMO/Al battery biased at $0 \mathrm{~V}$ (asreceived) and $0.5 \mathrm{~V}$ for 20 and $50 \mathrm{~min}$ [13].

Li was found to diffuse from LMO into $\mathrm{NbO}$ during the preparation of the specimen (analysis of the TERD spectrum of the as-received one). Under discharging and biasing conditions, $\mathrm{Ti}$ at $0.5 \mathrm{~V}$ (for 20 and $50 \mathrm{~min}$ to $\mathrm{Al}$ (sub) at earth potential) was studied. One can see from Figure 36 that the $\mathrm{Li}$ yields from $\mathrm{NbO}$ at $0.5 \mathrm{~V}$ (time: 20 and $50 \mathrm{~min}$ ) are reduced (than that of the as-received one under biasing conditions) and those from LMO are increased. To closely inspect the changes in Li, the normalized TERD spectra (normalized by $0 \mathrm{~V}$ ) are shown in Figure 37. The experiments were conducted under charging conditions. The samples were successively biased at $-3.2 \mathrm{~V}$ for $10 \mathrm{~min}$ and 40 min (Figure 38).

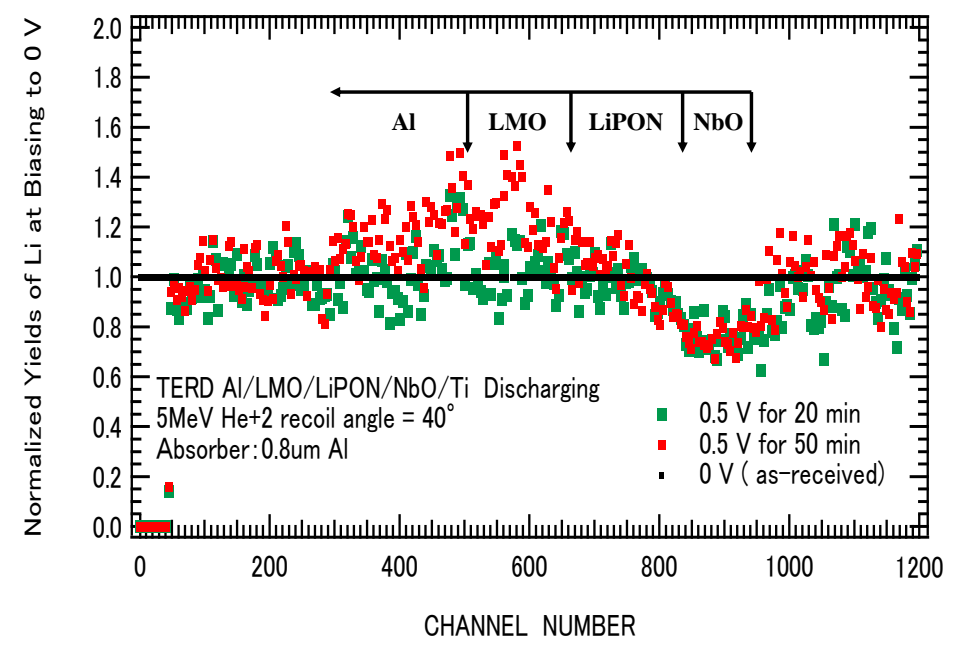

Figure 37 TERD spectral profiles of Ti/NbO/LiPON/LMO/Al biased at $0.5 \mathrm{~V}$ for 20 and 50 min, normalized by $0 \mathrm{~V}[13]$. 


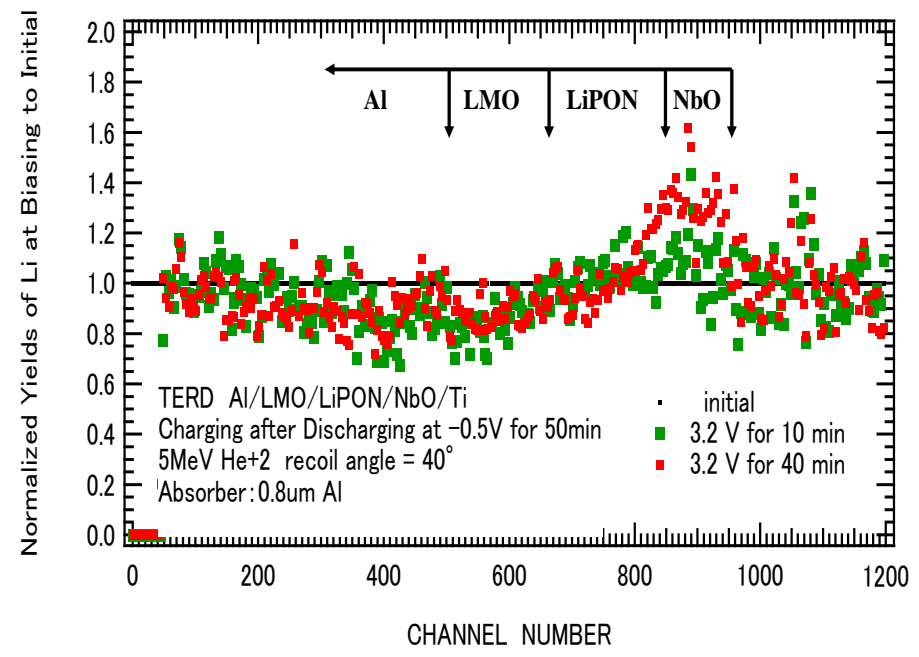

Figure 38 TERD spectral profiles of Ti/NbO/LiPON/LMO/Al biased at $3.2 \mathrm{~V}$ for 10 and 40 min, normalized by $0 \vee[13]$.

One can see clearly from Figure 37 that the Li yields from NbO decrease and those from LMO increase due to biasing at $0.5 \mathrm{~V}$ for $20 \mathrm{~min}$ and $0.5 \mathrm{~V}$ for $50 \mathrm{~min}$. The Li yields from Al also increase. The apparent large increase in the Li yields from Al is ascribed to the small amounts of Li diffusing into Al of the as-received specimen. One can also see that the Li yields from LiPON near NbO are reduced, and those near $\mathrm{LMO}$ get enriched. This can be attributed to the potential gradient. Furthermore, it can be seen from Figure 38 (obtained under charging conditions) that the Li yields from LMO and Al decrease, and those from NbO increase. The changes in the Li yields from LiPON correspond well with the observations made under biasing and discharging conditions. These results indicate that the movement of $\mathrm{Li}$ in the $\mathrm{Ti} / \mathrm{LMO} / \mathrm{LiPON} / \mathrm{NbO} / \mathrm{Al}$ battery is reversible (at least for one discharging and charging cycle), although $\mathrm{Li}$ is found to diffuse into the Al electrode. This can be attributed to the long loading time.

The battery fabricated using the Ti/NbO/LiPON/LMO/Ti substrate was studied by inserting a 2 $\mu \mathrm{m}$-thick $\mathrm{Al}$ absorber to exclude the overlapping of the impurity $\mathrm{H}$ peaks with the Li depth profile (of the battery of interest). The Ti substrate was biased at $0,1.5,2.0,2.7$, and $3.2 \mathrm{~V}$ to Ti at earth potential for $20 \mathrm{~min}$ under charging conditions. Following this, it was biased at $-1.0 \mathrm{~V}$ under discharging conditions. The TERD spectral profiles at approximately 300 - 800 channels were successfully separated from those of the impurity $\mathrm{H}$ at 1040 channels (Figure 39). The zoomed-in TERD spectral profiles at 2.0, 2.7, and 3.2 $\mathrm{V}$ under charging conditions and $-1.0 \mathrm{~V}$ under discharging conditions with the one at $0 \mathrm{~V}$ are closely compared in (a), (b), (c), and (d). The yields at $730-800$ channels, 500 - 730 channels, and 330 - 500 channels correspond to the layers of NbO, LiPON, and LMO, respectively. 

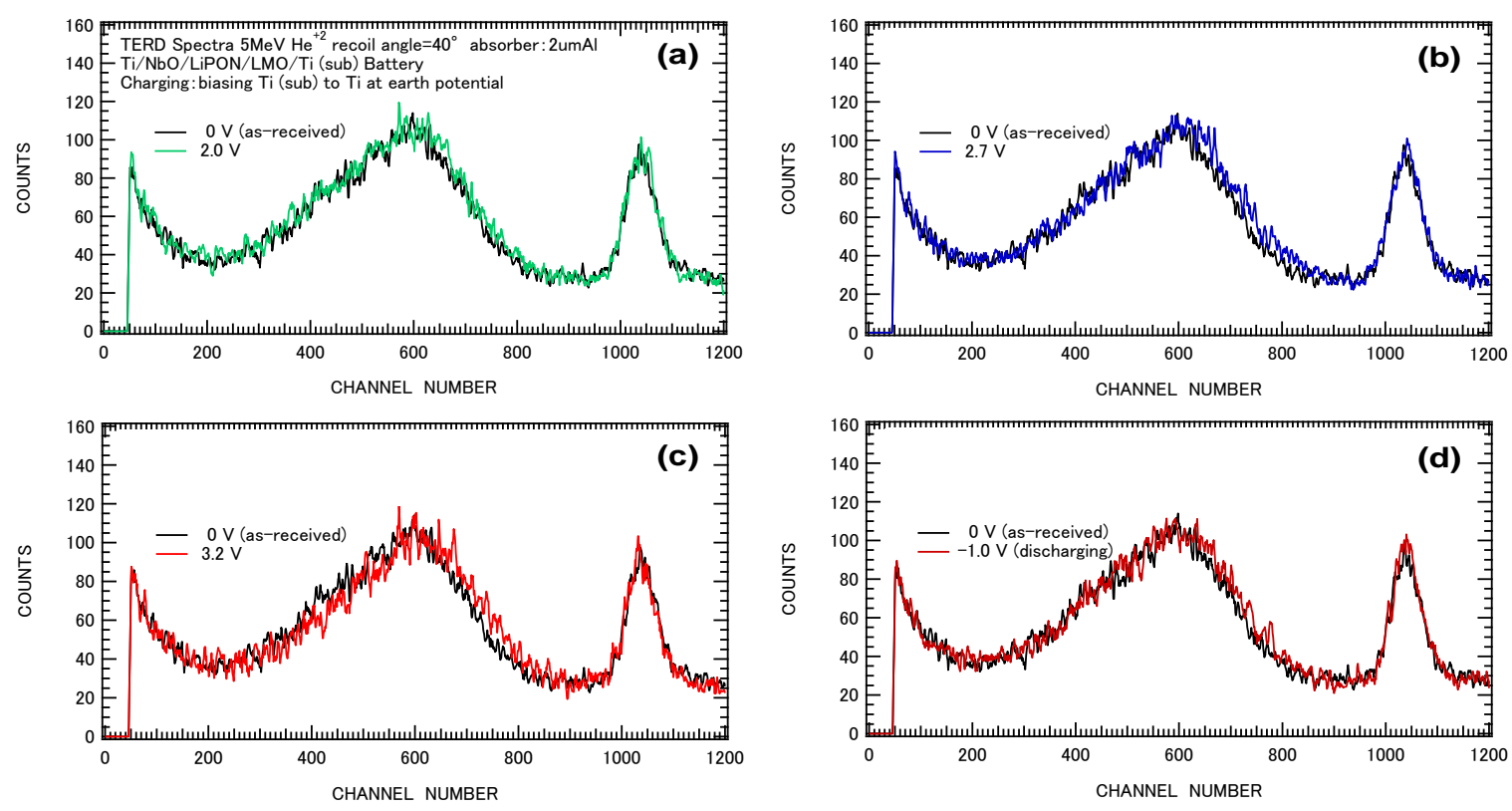

Figure 39 TERD spectral profiles of Ti/NbO/LiPON/LMO/Ti at: (a) $2.0 \mathrm{~V}$, (b) $2.7 \mathrm{~V}$, (c) 3.2 $\mathrm{V}$, and (d) $-1.0 \mathrm{~V}$ compared with that at $0 \mathrm{~V}[13]$.

It is seen from Figure 39 that the Li yields at $730-800$ channels increase with increasing bias voltage under charging conditions, whereas the yields of the system biased at $-1.0 \mathrm{~V}$ under discharging conditions decrease. It is seen from Figure 39 (a) that the Li yields from $\mathrm{NbO}$ at 730 800 channels increase at $2.0 \mathrm{~V}$. This indicates that the threshold bias voltage for charging is $\sim 2.0 \mathrm{~V}$.

To further study the changes in the Li depth profiles over the whole battery, the normalized Li yields at $0 \mathrm{~V}$ were calculated (Figure 40). The horizontal line at unity represents the as-received yields. To simplify the figure, the data at $2.0 \mathrm{~V}$ is omitted. One can also see from Figure 40 that the normalized yields from $\mathrm{NbO}$ increase at 2.7 and $3.2 \mathrm{~V}$ under charging and decrease at $-1.0 \mathrm{~V}$ under discharging conditions. The observation made under conditions of discharging (at $-1.0 \mathrm{~V}$ ) indicates that the battery is rechargeable.

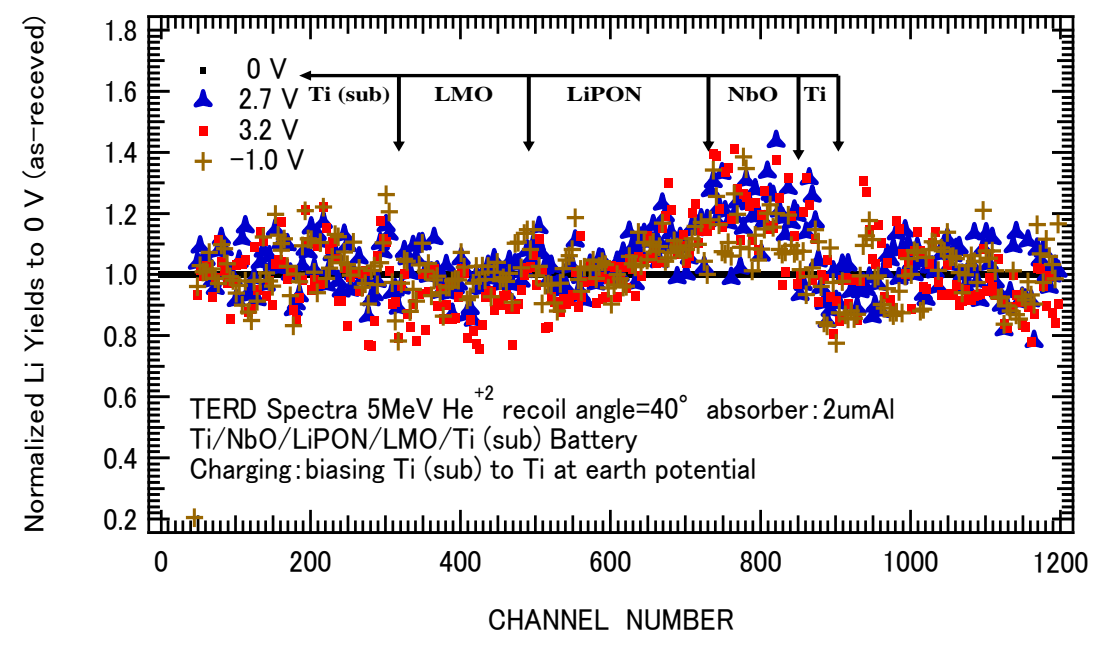

Figure 40 Normalized TERD spectral profiles at 2.7 and $3.2 \mathrm{~V}$ under charging conditions and $-1.0 \mathrm{~V}$ under discharging conditions (to $0 \mathrm{~V}$ ) [13]. 
On the other hand, it is found that although the yields from LiPON at 2.7 and $3.2 \mathrm{~V}$ are smaller than unity near LMO and larger near $\mathrm{NbO}$, the yields from LMO do not change. No changes in the latter case indicate that the increase in the $\mathrm{Li}$ yields from $\mathrm{NbO}$ under charging conditions can be attributed to the transport of $\mathrm{Li}$ (from the Ti substrate), which has diffused from LMO due to the temperature rise during the preparation of the specimen. Although the Li content in the Ti substrate is negligible, $\mathrm{Li}$ is considered to be uniformly distributed over the whole of the $1 \mu \mathrm{m}$-thick substrate. To confirm this, at least one charging and discharging cycle of the as-prepared specimen should be studied before conducting the ion beam analysis. Furthermore, the method to measure the Li depth profile in the absence of background yields caused by multiple scattering phenomena should be followed, as described below.

Finally, it is noted that the result obtained by studying Ti/NbO/LiPON/LMO/Ti (substrate) does not match with the result obtained by studying Ti/NbO/LiPON/LMO/Al (substrate), as described above, where the $\mathrm{Li}$ in $\mathrm{LMO}$ diffuses into $\mathrm{NbO}$ during the preparation of the specimen. This can be attributed to the fact that $\mathrm{Al}$ is more electro-positive than Ti.

\subsection{Development of the Recoil and Scattering Coincidence Method using the TERD Technique}

Recently, Mathayan et al., working at the Uppsala University, have demonstrated that the Li-Li and He-Li coincidence measurements [19] of the Ti/NbO/LiPON/LMO/Ti (substrate) battery in the presence of the $8 \mathrm{MeV} \mathrm{Li}$-ion and $4.5 \mathrm{MeV}$ He ion beams can be successfully used to determine the total $\mathrm{Li}$ depth profile in the absence of background yields caused by the multiple scattering phenomena, which originate in the $\mathrm{H}-\mathrm{H}$ coincidence method. This method can be used to detect hydrogen in the solid target [20]. Here, the data on the He-Li coincidence measurement in the presence of the $4 \mathrm{MeV}$ He beam of the as-prepared Ti/NbO/LiPON/LMO/Ti (substrate) battery have been presented.

A geometrical schematic of He-Li coincidence measurement, the experimental results on the 3D coincidence image, and the TERD energy spectrum of Li are shown in Figure 41 and Figure 42. In Figure 41 , the detector ssd 2 at $45^{\circ}$ is used to measure the recoil of Li. The detector ssd 1 is not used to measure the collision partner of the scattered He, where both the detector angles can be computed from the scattering angle $\theta$ and recoiling angle $\phi$. The relation is presented in the equation representing elastic collision: $\phi=\pi / 2-\xi / 2$ and $\tan \theta=\left(M_{2} \sin \xi\right) /\left(M_{1}+M_{2} \cos \xi\right)$. Here, $\xi$ is the scattering angle in the center of the mass system.

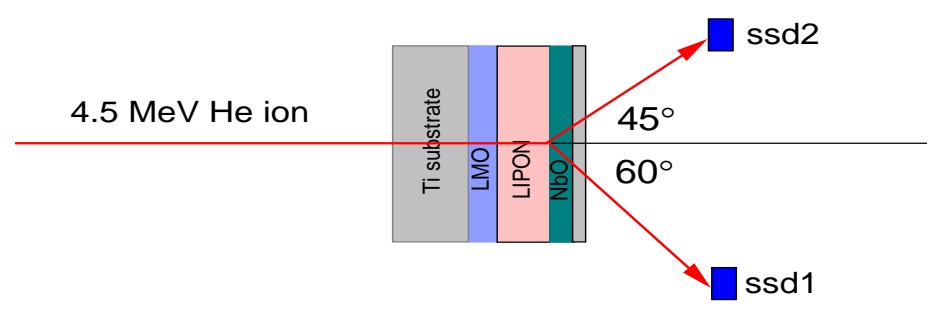

Figure 41 Geometrical schematic of the He-Li coincidence measurement of the $\mathrm{Ti} / \mathrm{NbO} / \mathrm{LiPON} / \mathrm{LMO} / \mathrm{Ti}$ (substrate) battery. The Detector ssd 2 at $45^{\circ}$ is used to study Li, and the detector $\operatorname{ssd} 1$ at $60^{\circ}$ is used to study He [20]. 

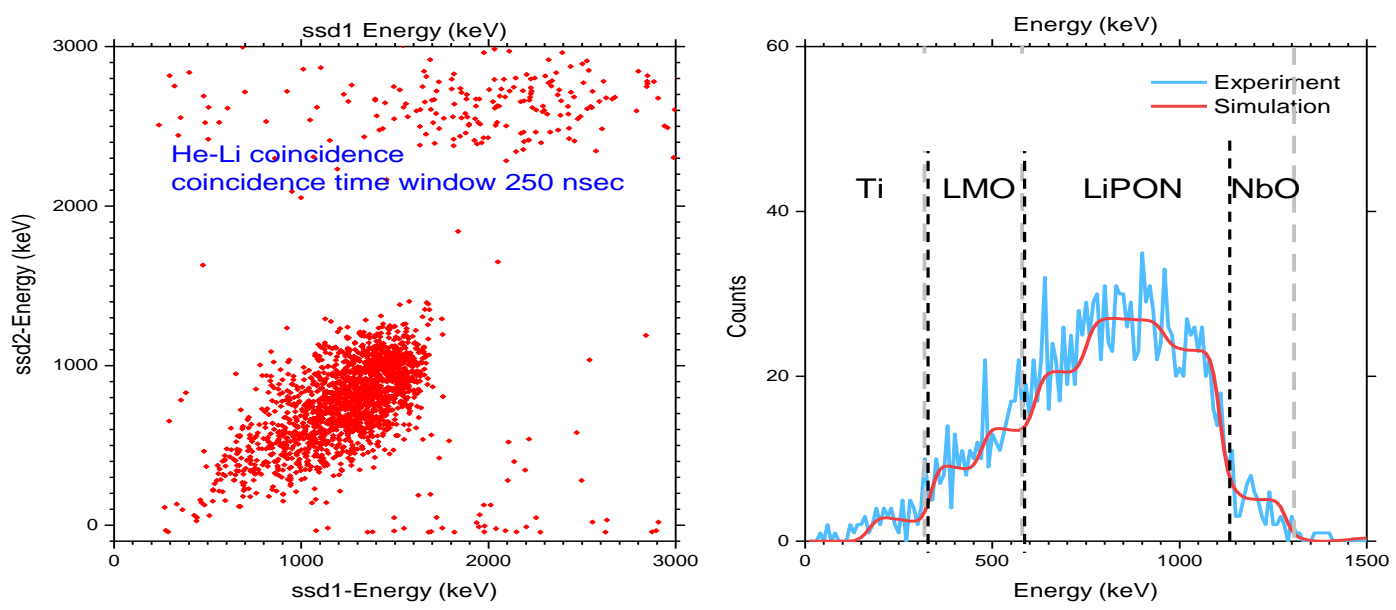

Figure 42 (left) 3D He-Li coincidence image and (right) TERD Li energy spectrum [20].

It is seen from the 3D image in Figure 42 (left) that there are no background yields around the coincidence yields. It is also seen from Figure 42 (right) that the TERD spectrum of Li, which has been obtained from the projection of the vertical axis, does not exhibit background yields. This is contrary to the observation made for the one shown previously in Figure 36 (Section 4-2). Thus, one can easily distinguish the Li yields in each film. Analysis of the TERD energy spectrum has revealed that $\mathrm{Li}$ in $\mathrm{LMO}$ diffuses into $\mathrm{Ti}$ (substrate) and $\mathrm{NbO}$ during the preparation of the specimen. It has also been observed that the Li content can be determined using the TERD technique.

The results obtained by studying the $\mathrm{Ti} / \mathrm{NbO} / \mathrm{LiPON} / \mathrm{LMO} / \mathrm{Ti}$ substrate battery have been presented for the reaction between $\mathrm{Li}$ and Ti electrodes under conditions of over-charging. Recently, V. Mathayan has used the recoil-scatter coincidence method of the TERD technique to study this phenomenon [19].

The He-Li coincidence TERD energy spectra were recorded under biasing conditions at 0 and $5 \mathrm{~V}$ for $45 \mathrm{~min}$ and $-5 \mathrm{~V}$ for $75 \mathrm{~min}$ (Figure 43). The top Ti surface and the interfaces between each film in the battery are indicated by dotted lines. One can easily distinguish the films (Figure 43), even though the yield counts at the vertical axis are not significantly large. It is seen from Figure 43 that $\mathrm{Li}$ in the as-prepared specimen at $0 \mathrm{~V}$ diffuses into $\mathrm{NbO}$, and large amounts of $\mathrm{Li}$ are charged from LMO into NbO. Diffusion into the top Ti electrode under biasing conditions at $5 \mathrm{~V}$ (time: $45 \mathrm{~min}$ ) corresponds to the condition of over-charging. The results agree well with the results described previously in Section 5-2. It is also seen that upon discharging at -5 V (time: $75 \mathrm{~min}$ ), the Li charged into $\mathrm{NbO}$ does not return to LMO and LiPON. A small peak at approximately 340 channel indicates the segregation of $\mathrm{Li}$ at the Ti surface. This fact indicates that overcharging creates a barrier for $\mathrm{Li}$ movement at the interface of LiPON and NbO. Although the battery's performance is not too good, direct observation of the anomaly from the TERD spectral profile is a wonderful outcome of the HeLi coincidence measurement method. On the other hand, in the profile presented in Section 5-2, the peak cannot be detected because of poor energy resolution, which can be attributed to the large energy straggling of the incident $\mathrm{O}$ ions $(9 \mathrm{MeV})$. 


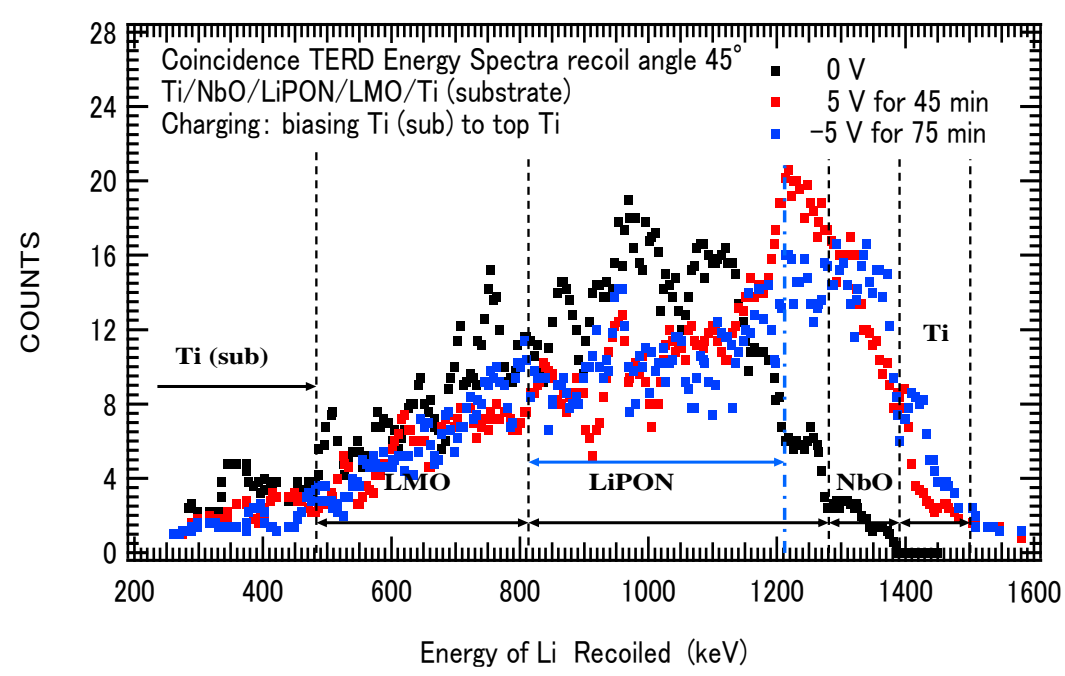

Figure $43 \mathrm{He}$-Li coincidence TERD energy spectral profiles recorded at $0 \mathrm{~V}$ and $5 \mathrm{~V}$ for 45 min and-5 $\mathrm{V}$ for $75 \mathrm{~min}$ in the presence of the $4 \mathrm{MeV} \mathrm{He} \mathrm{H}^{+2}$ beam.

\section{Summary}

In this review, various studies on the Li depth profiles of the metal/electrolyte/metal capacitors and $\mathrm{Au} / \mathrm{LCO} / \mathrm{LATP} / \mathrm{Pt}$, LCO/LiPON/Si, and LMO/LiPON/NbO batteries with different metal electrodes at both sides under bias using the in-situ ERD technique with the $9 \mathrm{MeV}^{+4}$ ion beam have been reported. The TERD technique with the $5 \mathrm{MeV} \mathrm{He}^{+2}$ ion beam was also used for the studies.

The studies on the $\mathrm{Cu}(\mathrm{Al}) / \mathrm{LiPON} / \mathrm{Cu}$ (Al) and Au/LATP/Au capacitors have been described in Section 3. It has been shown that when the top $\mathrm{Cu}$ was biased at $-3 \mathrm{~V}$ to the bottom $\mathrm{Cu} / \mathrm{Ti}$ of $\mathrm{Cu} / \mathrm{LiPON} / \mathrm{Cu} / \mathrm{Ti} / \mathrm{SiO}_{2}$, $\mathrm{Li}$ is segregated at the top $\mathrm{Cu}$ surface. This indicates that the voltage of $-3 \mathrm{~V}$ causes an overload. The changes in the $\mathrm{Li}$ depth profiles were determined under steady-state conditions below $2 \mathrm{~V}$. The results show that $\mathrm{Li}$ in LiPON is enriched near the metal biased negatively. It is depleted near the metal biased positively, and the enrichment and depletion of Li at both sides are asymmetrical. This has been explained in terms of the difference between the Fermi energies, which can be attributed to the buffer layer of Ti, the Fermi energy of which is higher than that of and $\mathrm{Cu}$. A symmetrical change is observed for Au/LATP/Au. It has also been shown that the Li depth profile calculated on the model of hopping diffusion for $\mathrm{Li}$ ions under potential gradient corresponds well with the experimentally obtained one. The fraction of Li ions transported under the influence of a potential gradient in the electrolyte is less than unity. The Li atoms diffuse due to the concentration gradient. The diffusion occurs in the direction opposite to the observed under the influence of a potential gradient.

The studies on the Au/LCO/LATP/Pt battery have been presented in Section 4. It has been shown that the threshold bias voltage for charging from LCO into LATP near Pt was $1.3 \mathrm{~V}$. The Li content in LCO is gradually and uniformly reduced over the whole depth. The final content was $\sim 0.3$ when the voltage was increased beyond $1.3 \mathrm{~V}$ (till $2.4 \mathrm{~V}$ ). The relation between Li composition and the bias voltage agrees well with the relation obtained previously using the other techniques. When the system is discharged, an anomaly is observed. Li is not enriched but further reduced under biasing conditions at $0.5 \mathrm{~V}$ (time: $25 \mathrm{~min}$ ). Further biasing (discharging) at 1.0, 1.5, and $2.2 \mathrm{~V}$ for $50 \mathrm{~min}$ results in the uniform enrichment of $\mathrm{Li}$ in LCO over the whole depth. The anomaly has been 
explained in terms of the formation of a barrier (around $1.0 \mathrm{~V}$ high) at the interface between LCO and LATP due to the over-reduction of Li due to the charging. The fact that the transport fraction of $\mathrm{Li}$ ions is less than unity induces the counter flow of the $\mathrm{Li}$ atom. However, continuous biasing indicates that the battery is rechargeable. When $\mathrm{Li}$ is reduced from LCO (to 0.6 by charging), $\mathrm{Li}$ is rapidly transported back from LATP to LCO under biasing and discharging conditions at $1.0 \mathrm{~V}$. No anomaly is observed.

For the Ti/NbO/LiPON/LMO/Ti/Si (substrate) battery, it has been shown that Li diffuses from LMO to NbO during its preparation and Li charged in $\mathrm{NbO}$ exhibits a diffusing slope (in the direction from LiPON to Ti electrode). Moreover, it diffuses into the Ti electrode under conditions of overcharging. Upon subsequent discharging, Li charged into $\mathrm{NbO}$, and subsequently, the diffusion in the $\mathrm{Ti}$ electrode was reduced. For the Ti/LMO/LiPON/NbO/Ti/Si (substrate) battery, Li charged into NbO from $L M O$ returns to $L M O$. It diffuses into the top Ti unit and segregates at the surface due to overcharging. Such a large difference in the Li movement is attributed to the Schottky barrier, which can be attributed to the difference in the Fermi levels at the interfaces of $\mathrm{LMO} / \mathrm{Ti}$ and $\mathrm{NbO} / \mathrm{Ti}$ on the $\mathrm{Si}$ substrate.

In Section 5, the changes in the total Li depth profiles were determined using the high depth resolution TERD technique in the presence of the $5 \mathrm{MeV} \mathrm{He}^{+2}$ beam.

For the Au/Si/LiPON/LCO/Au/Al (sub) battery, it has been shown that Li that has diffused into Si from LCO during its preparation returns to LCO and diffuses into $\mathrm{Al}$ (sub) through Au under biasing conditions at $1.3 \mathrm{~V}$ and $2.0 \mathrm{~V}$. On the other hand, in the Ni/Si/LiPON/LCO/Pt/Ti/SiN(sub) battery, Li that has diffused into $\mathrm{Si}$ diffuses back into $\mathrm{Ni}$ in the follow direction of potential gradient due to biasing at $2.0 \mathrm{~V}$ and $2.8 \mathrm{~V}$. The difference between the transport of $\mathrm{Li}$ in both the batteries under conditions of over-charging has been explained in terms of the difference in the Fermi energies of the metal electrodes at both sides. The height of the Schottky barrier at the interfaces of Au/Si and $\mathrm{Ni} / \mathrm{Si}$ was influenced by the transport fraction of the Li-ions (less than unity) under the influence of a potential gradient. The Li atoms are transported under the influence of the concentration gradient in the counter direction.

In Ti/NbO/LiPON/LMO batteries ( $\mathrm{LMO}=\mathrm{LiMn}_{2} \mathrm{O}_{4}, \quad \mathrm{LiPON}=\mathrm{Li}_{3.3} \mathrm{PO}_{3.8} \mathrm{~N}_{0.2}$, and $\left.\mathrm{NbO}=\mathrm{Nb}_{2} \mathrm{O}_{5}\right)$ prepared on self-supporting $\mathrm{Al}$ and Ti substrates, it has been shown that in Ti/NbO/LiPON/LMO/Al and Ti/NbO/LiPON/LMO/Ti battery, the movement of Li between NbO and LMO under discharging and charging conditions is reversible. It has also been observed that the battery is rechargeable. On the other hand, during the preparation of the Ti/NbO/LiPON/LMO/Ti battery, Li diffuses from LMO into $\mathrm{Ti}$ (sub) which is much thicker than the top Ti. In the Ti/NbO/LiPON/LMO/Al battery, Li diffuses from LMO and Al (sub), which is more electro-positive than the top Ti electrode.

In Section 5-3, the recoil-scatter coincidence method in the TERD technique in the presence of the $4 \mathrm{MeV} \mathrm{He}^{+2}$ beam (developed recently) has been introduced and preliminary results have been presented. The results reveal that the Li depth profiles in the absence of background yields could be determined by the process of multiple scattering.

Finally, Li transport and its reaction with metal electrodes in different batteries under anomalous conditions have been studied. The anomalies were positively induced by over-biasing under charging and discharging conditions (Table I). The work functions of Pt, $\mathrm{Au}, \mathrm{Ni}, \mathrm{Ti}$, and $\mathrm{Al}$ are 5.64, $5.38,5.20,4.33$, and $4.17 \mathrm{eV}$ [21], respectively. 
Table 1 Comparison of Li transport and reaction in different batteries under anomalous conditions induced by over-biasing under charging and discharging conditions.

\begin{tabular}{|c|c|c|c|}
\hline \multirow{2}{*}{ techniques } & \multirow{2}{*}{ batteries } & \multicolumn{2}{|l|}{ Li transport } \\
\hline & & Charging & Discharging \\
\hline ERD & $\mathrm{Pt} / \mathrm{LATP} / \mathrm{LCO} / \mathrm{Au}$ & $\begin{array}{l}\text { barrier formation at } \\
\mathrm{LCO} / \mathrm{Au} \text { interface at } \\
2.4 \mathrm{~V}\end{array}$ & $\begin{array}{l}\text { returns from } L C O \text { to } P t \\
\text { at }-0.5 \mathrm{~V} \\
\text { returns from } L C O \text { to } P t \\
\text { at }-0.5 \mathrm{~V}\end{array}$ \\
\hline TERD & $\mathrm{Au} / \mathrm{Si} / \mathrm{LiPON} / \mathrm{LCO} / \mathrm{Au} / \mathrm{Al}$ & $\begin{array}{l}\text { returns from Si to } \mathrm{LCO} \\
\text { and diffuses into } \mathrm{Au} / \mathrm{Al} \\
\text { at } 2.8 \mathrm{~V}\end{array}$ & no data \\
\hline TERD & $\mathrm{Ni} / \mathrm{Si} / \mathrm{LiPON} / \mathrm{LCO} / \mathrm{Pt} / \mathrm{Ti}$ & $\begin{array}{l}\text { not returns, but } \\
\text { diffuses into } \mathrm{Ni} \text { at } 2.8 \mathrm{~V} \\
\text { and } 3.8 \mathrm{~V}\end{array}$ & no data \\
\hline ERD & Ti/NbO/LiPON/LMO/Ti* & $\begin{array}{l}\text { saturates in } \mathrm{NbO} \text { at } \\
4.5 \mathrm{~V} \text { and diffuses into } \\
\mathrm{Ti} \text { at } 5.5 \mathrm{~V}\end{array}$ & $\begin{array}{l}\text { no reduction in } \mathrm{NbO} \\
\text { and returns from } \mathrm{Ti} \text { to } \\
\mathrm{LMO} \text { at } 2.5 \mathrm{~V}\end{array}$ \\
\hline TERD & Ti/NbO/LiPON/LMO/Al & $\begin{array}{l}\text { saturates in } \mathrm{NbO} \text { and } \\
\text { returns into } \mathrm{Al} \text { via } \mathrm{LMO}\end{array}$ & $\begin{array}{l}\text { reduction at } 0.5 \mathrm{~V} \text { for } 50 \\
\text { min and diffuses into } \mathrm{Al} \\
\text { via LMO }\end{array}$ \\
\hline $\begin{array}{l}\text { Coincidence } \\
\text { TERD }\end{array}$ & Ti/NbO/LiPON/LMO/Ti* & $\begin{array}{l}\text { saturates in } \mathrm{NbO} \text { and } \\
\text { returns up to } \mathrm{Ti}^{*} \text { via } \\
\mathrm{LMO} \text { at } 5 \mathrm{eV}\end{array}$ & $\begin{array}{l}\text { saturates in } \mathrm{LMO} \text { and } \\
\text { diffuses into Ti via } \mathrm{NbO} \\
\text { at }-5 \mathrm{eV}\end{array}$ \\
\hline
\end{tabular}

It is seen from Table 1 that except for the Pt/LAPT/LCO/Au battery where the capacity of Li in the anode formed at the interface of Pt and LAPT is sufficiently large than that in LCO, the over-flow of $\mathrm{Li}$ in anode takes place in the batteries of Si/LiPON/LCO and NbO/LiPON/LMO. Here, the capacity of $\mathrm{Li}$ in anode was limited by their thicknesses. In general, the batteries are well designed so that the capacity of $\mathrm{Li}$ in the anode is larger than that in the cathode. Therefore, the balance between the $\mathrm{Li}$ capacities in the anode and cathode is lost during the preparation of the battery specimen. As shown above, the presence of hydrogen has been observed in the anode, separator, and cathode of all the as-prepared specimens. Thus, the origin of the loss of the balance may be attributed to the evolution of hydrogen during the preparation of the specimen following the conventional magnetron sputter method. They might occupy the sites of $\mathrm{Li}$ in the anode. The reduction of the Li capacities at the anode seems to be qualitatively larger than that in the cathode.

Although the Li composition near the interface attains saturation, the reaction of Pt and Au with Li was not observed, which can be attributed to their high work functions. It is important to fabricate batteries in which the capacity of Li in the anode is not limited under any condition. Hence, further 
systematic studies on the system with the metal electrode with low work function at both sides should be conducted. On the other hand, to avoid the reaction between Li and the metal electrodes under accidental over-charging conditions, further studies on NbO/LiPON/LMO with high work function metal electrodes (such as Pt and $\mathrm{Au}$ ) should also be conducted.

\section{Conclusive Remark}

The changes in the Li depth profiles of the metal/electrolyte/metal capacitors and solid-state batteries have been critically reviewed on the basis of the data obtained in-situ under bias using the ERD, TERD, coincidence TERD, and a combination of these techniques with the RBS technique in the presence of the MeV ion beam. During each experiment, the specimen was shifted toward the incident ions to minimize the damages caused due to irradiation.

Hydrogen is involved in all the specimens prepared following the conventional magnetron sputter and laser ablation deposition methods. When the capacitors were studied, it was found that the transport fraction of the Li ions in the LATP and LiPON electrolytes under biasing conditions was less than unity and the Li atoms diffused in the direction opposite to the direction of biasing. It was also found that the batteries were rechargeable.

Furthermore, changes in the Li depth profiles over the whole battery under anomalous conditions (positively induced by over-biasing under charging and discharging conditions) have been determined to investigate the reactions of Li with the metal electrodes. For the Au/LCO/LATP/Pt battery where the capacity of Li formed at the interface between LATP and Pt is significantly larger than that in LCO (post over-biasing), no discharge under biasing at $0.5 \mathrm{~V}$ was observed due to the formation of a barrier at the LATP/LCO interface.

For the Si/LiPON/LCO and NbO/LiPON/LMO batteries where the capacity of Li is limited at the anodes by their thicknesses, over-charging and strong reactions of Li with the metal electrodes are observed (Table I). In general, the capacities of the Li at the anode and cathode in the battery are well balanced and influenced by its design. The over-charging observed is ascribed to the imbalance brought about during the preparation of the specimens, namely the reduction in the capacity of Li in the anode induced by a sizable amount of hydrogen impurity, which has been observed in all the specimens. Therefore, to exclude such over-charging and avoid the involution of impurity hydrogen during sample preparation, it is important to realize the realistic usage of the battery.

Finally, further systematic measurements of the hydrogen-free specimen are required.

\section{Highlights}

This review is a summary of the in-situ operand experimental data on solid-state Li-ion batteries obtained using the combined techniques of ERD, TERD, and RBS in the presence of MeV ion beams.

The metal/electrolyte/metal capacitors were studied and it was found that the transport fraction of the Li ions in the electrolyte under biasing conditions is less than unity. It has also been observed that the Li atoms diffuse in the direction that is opposite to the direction of biasing. This plays an important role in the reactions of Li with metal electrodes under anomalous battery conditions induced by over-biasing. The solid-state Au/LCO/LATP/Pt, Si/LiPON/LCO, and NbO/LiPON/LMO batteries with different electrodes at both sides are rechargeable.

The anomalies observed under conditions of over-charging have been brought about by overbiasing performed positively under charging and discharging conditions. Under these conditions, 
strong reactions of Li with both the metal electrodes are observed. The anomaly can be attributed to the imbalance between the capacities of $\mathrm{Li}$ at the anode and cathode, which is caused by the presence of a sizable amount of hydrogen impurities (involved during sample preparation). Finally, the method for the preparation of the hydrogen-free battery is proposed.

\section{Outlook}

In this review, the in-situ experimental data on the solid-state Li ion batteries obtained using the $\mathrm{MeV}$ ion beam analysis have been summarized.

It reveals that the hydrogen involved during sample preparation may bring about reactions between $\mathrm{Li}$ and metal electrodes due to over-biasing on charging. It is difficult to detect hydrogen in general. It is emphasized that the method of preparation of hydrogen-free battery should be developed to avoid over-charging, hinder the reduction in the amounts of movable Li ions, and extend the lifetime of the battery by reducing the interaction of Li with the metal electrodes (extent of corrosion should be reduced).

The target-materials for magnetron sputter include considerably large amounts of hydrogen because their elemental compositions are very similar to the hydrogen storing Li-containing oxide ceramics absorbing hydrogen from water vapor at room temperature [22]. Trace levels of water vapor included in the working gases of magnetron sputter, which are accumulated in the chamber during its long-term operation [23], also assist the process. Hydrogen-free specimen will be realized by utilizing both the sintered target and liquid nitrogen traps from which hydrogen and water vapor can be eliminated following the pre-annealing and the adsorption processes, respectively.

\section{Acknowledgments}

These works were partly supported by Grant-in-Aid, Scientific Research (C25420762), JSPS and Global Research Center for Environment and Energy based on Nano-materials Science (GREEN), NIMS. They were also performed under the valuable co-operative program of Tandem Accelerator both at the Quantum Energy Science and Engineering Education and Research Center, Kyoto University, at the Wakasawan Energy Research Center and at the Ängström Laboratory, Uppsala University, Sweden. We are sincerely grateful to Professor Yasutoshi Iriyama for valuable discussions at the GREEN program and a number of undergraduate, MC and DC graduate students in his laboratory for preparation of the battery specimens and technical support on electro-chemical measurements, Graduate School of Engineering, Nagoya University. We are also partly indebted to Dr. Rongbin Ye, Department of Innovation Systems Engineering, Iwate University for preparation of the battery specimen. Moreover, we are grateful to Professor Akio Itoh for giving chance of the cooperative research, to Drs. Hidetsugu Tsuchida and Takuya Majima for joining the experiments and to Mr. Yoshitaka Sasaki for his friendly technical support at the Quantum Science and Engineering Center, Kyoto University, to Dr Koutaku Suzuki at the Wakasawan Energy Research Center and to Drs Daniel Premetzhofer, Marucos Moro, Maruicio Sortica and Vairavel Mathayan at the Ängström Laboratory, Uppsala University, Sweden for joining the collaborative experiments.

\section{Author Contributions}

K. Morita: Supervision, Conceptualization, Investigation, Data curation, Writing - original draft. 
B. Tsuchiya: Investigation, Data curation, review \& editing.

\section{Competing Interests}

The authors have declared that no competing interests exist.

\section{References}

1. Itoh $\mathrm{M}$, Inaguma $\mathrm{Y}$, Jung $\mathrm{WH}$, Chen $\mathrm{L}$, Nakamura $\mathrm{T}$. High lithium-ion conductivity in the perovskite-type compounds $\mathrm{Ln}_{12} \mathrm{Li}_{12} \mathrm{TiO}_{3}$ ( $\mathrm{Ln}=\mathrm{La}$, $\mathrm{Pr}, \mathrm{Nd}$, Sm). Solid State Ion. 1994; 70-71: 203207.

2. Kanno R, Maruyama M. Lithium Ionic Conductor Thio-LISICON: The $\mathrm{Li}_{2} \mathrm{~S} \mathrm{GeS}_{2} \mathrm{P}_{2} \mathrm{~S}_{5}$ System. J Electrochem Soc. 2001; 148: A742.

3. Mizno F, Hayashi A, Tadanaga K, Tatsumisago M. New, Highly Ion-Conductive Crystals Precipitated from Li2S-P2S5 Glasses. Adv Mater. 2005; 17: 918-921.

4. Yamamoto K, Iriyama Y, Asaka T, Hirayama T, Fujita H, Fisher CAJ, et al. Dynamic visualization of the electric potential in an All-Solid-State rechargeable lithium battery. Angew Chem Int Ed. 2010; 49: 4414-4417.

5. Oudenhoven JFM, Labohm F, Mulder M, Niesen RAH, Mulder FM, Notten PHL. In Situ Neutron Depth Profiling: A Powerful Method to Probe Lithium Transport in Micro-Batteries. Adv Mater. 2011; 23: 4103-4106.

6. Tsuchiya B, Morita K, Nagata S, Kato T, Iriyama Y, Tsuchida H, et al. Dynamic measurements of Li depth profiles in a Li-ion battery system under charging condition by means of ERD and RBS techniques. Surf Interface Anal. 2014; 46: 1187-1191.

7. Morita K, Tsuchiya B, Oonishi J, Yamamoto T, Iriyama Y, Tsuchida H, et al. High-Resolution Li Depth Profiling of Solid-State Li Ion Battery by Means of TERD Technique with High Energy Light lons, Nucl. Instrum. Methods Phys Res B. 2018; 427: 30-33.

8. Morita K, Tsuchiya B, Oonishi J, Yamamoto T, Iriyama Y, Tsuchida H, et al. K. Morita, B. Tsuchiya, J. Oonishi, N. Mitsukuchi, T. Yamamoto, Y. Iriyama, H. Tsuchida and T. Majima. High Resolution In-situ Depth Profiling of Thin Films Stacked Li Ion Batteries under Charging Conditions by Means of TERD and RBS Techniques with $5 \mathrm{MeV} \mathrm{He}^{+2}$ Ion Beam, Nucl. Instrum. Methods Phys Res B. 2018; 436: 8-12.

9. Tsuchiya B, Oonishi J, Sasaki Y, Yamamoto T, Yamamoto Y, Motoyama M, et al. Solid-StateBattery interface: In situ direct lithium distribution analysis around interfaces in an All-SolidState rechargeable lithium battery by combined Ion-Beam method. Adv Mater Interfaces. 2019; 6: 1970090.

10. Morita K, Tsuchiya B, Tsuchida H, Majima T. Li depth profiles of metal/Li-electrolyte/metal capacitors under biasing studied by means of $\mathrm{MeV}$ ion beam analysis techniques. Solid State Ion. 2020; 344: 115085.

11. Morita K, Tsuchiya B, Tsuchida H, Majima T. Change in Li depth profiles of Au/LCO/mixed LATPLAGP/Pt battery under discharging studied by ion beam analysis techniques with $9 \mathrm{MeV}$ O4+ ions. Solid State Ion. 2020; 344: 115135.

12. Morita K, Tsuchiya B, Ye R, Tsuchida H, Majima T., Dynamic Behaviour of Li Depth Profiles in Solid State Li Ion Battery under Charging and Discharging by Means of ERD and RBS Techniques, Solid State Ion. (2021) accepted for publication. 
13. Morita K, Tsuchiya B, Ye R, Tsuchida H, Majima T. In-situ total Li depth profiling of solid-state Li ion batteries under charging and discharging by means of transmission elastic recoil detection analysis with $5 \mathrm{MeV} \mathrm{He2+} \mathrm{ions.} \mathrm{Nucl} \mathrm{Instrum} \mathrm{Methods} \mathrm{Phys} \mathrm{Res} \mathrm{B.} \mathrm{2020;} \mathrm{479:} \mathrm{249-253.}$

14. L'Ecuyer J, Brassard C, Cardinal C, Chanbbal J, Deschenes L, Labrie JP, et al. An accurate and sensitive method for the determination of the depth distribution of light elements in heavy materials. J Appl Phys. 1976; 47: 381-382.

15. Iriyama $Y$, Inaba M, Abe T, Ogumi Z. Preparation of $c$-axis oriented thin films of LiCoO2 by pulsed laser deposition and their electrochemical properties. J Power Sources 2001; 94: 175-182.

16. Ye R, Yanami Y, Ohta $\mathrm{K}$, Baba M. Electrochemical properties of amorphous $\mathrm{Nb}_{2} \mathrm{O}_{5}$ thin film and its application to rechargeable thin film lithium-ion batteries. ECS trans. 2016; 73: 49-55

17. Foti G, Mayer J, Rimini R. Backscattering Spectrometry. In: Ion beam handbook for material analysis. New York: Academic Press Inc; 1977.

18. Braun S, Yada C, Latz A. Thermodynamically consistent model for Space-Charge-Layer formation in a solid electrolyte. J Phys Chem C. 2015; 119: 22281-22288.

19. Yazami R. Thermodynamics of electrode materials for Lithium-Ion batteries. In: Lithium-lon Rechargeable Batteries: Materials, Technology, and New Applications. Wiley-VCH; 2009. 67102.

20. Mathayan V, Moro MV, Morita K, Tsuchiya B, Ye R, Baba M. In-operando observation of Li depth distribution and Li transport in thin film Li ion batteries. Appl Phys Lett. 2020; 117: 023902.

21. CRC. CRC Handbook of Chemistry and Physics. 78th Ed. New York: CRC Press; 1998.

22. Morita K, Tsuchiya B. A mechanism for water splitting and hydrogen absorbing functions of metal-oxide layered hydrogen storage materials studied by means of ion beam analysis. Surf Interface Anal. 2014; 46: 113-127.

23. Tsuchiya B, Morita K. Retention and re-emission of hydrogen in beryllium studied by the ERD technique. J Nucl. Mater. 1996; 233-237: 898-901.

24. Martinsson BG, Kristiansson P. A high-sensitivity method for hydrogen analysis in thin targets. Nucl. Instru. Methods Phys Res B. 1993; 82: 589-599.

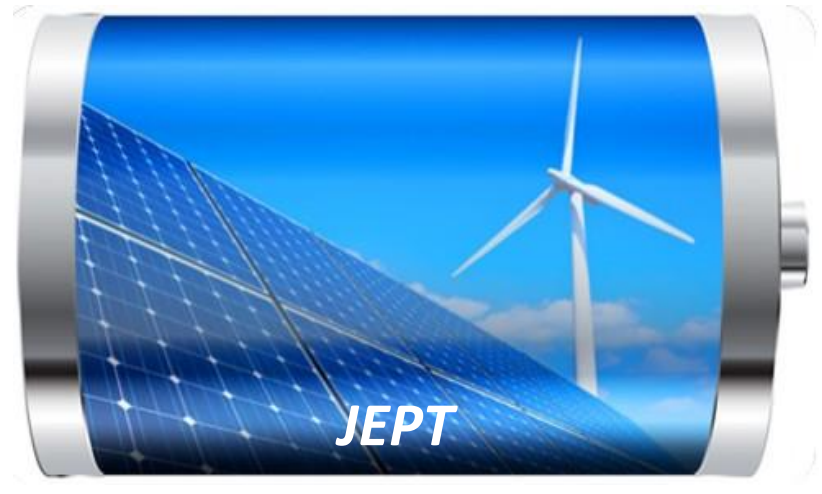

Enjoy JEPT by:

1. Submitting a manuscript

2. Joining in volunteer reviewer bank

3. Joining Editorial Board

4. Guest editing a special issue

For more details, please visit:

http://www.lidsen.com/journal/jept 